\title{
ÉTALE MOTIVIC COHOMOLOGY AND ALGEBRAIC CYCLES
}

\author{
ANDREAS ROSENSCHON ${ }^{1}$ AND V. SRINIVAS ${ }^{2}$ \\ ${ }^{1}$ Mathematisches Institut, Ludwigs Maximilians Universität, München, Germany \\ (axr@math.lmu.de) \\ ${ }^{2}$ School of Mathematics, Tata Institute of Fundamental Research, Mumbai, India \\ (srinivas@math.tifr.res.in)
}

(Received 26 May 2014; revised 31 October 2014; accepted 1 November 2014; first published online 24 November 2014)

\begin{abstract}
We consider étale motivic or Lichtenbaum cohomology and its relation to algebraic cycles. We give an geometric interpretation of Lichtenbaum cohomology and use it to show that the usual integral cycle maps extend to maps on integral Lichtenbaum cohomology. We also show that Lichtenbaum cohomology, in contrast to the usual motivic cohomology, compares well with integral cohomology theories. For example, we formulate integral étale versions of the Hodge and the Tate conjecture, and show that these are equivalent to the usual rational conjectures.
\end{abstract}

Keywords: motivic cohomology; algebraic cycles

2010 Mathematics subject classification: Primary 19E15

Secondary 19E20

\section{Introduction}

Let $X$ be a smooth quasi-projective variety over a field $k$. We interpret the motivic cohomology of $X$ with coefficients in an abelian group $A$ as the hypercohomology groups

$$
\mathrm{H}_{\mathrm{M}}^{m}(X, A(n))=\mathbb{H}_{\mathrm{Zar}}^{m}\left(X,\left(z^{n}(-, \bullet) \otimes A\right)[-2 n]\right),
$$

where $z^{n}(-, \bullet)$ is the complex of Zariski sheaves given by Bloch's cycle complex [6]. In particular, $\mathrm{H}_{\mathrm{M}}^{2 n}(X, \mathbb{Z}(n))=\mathrm{CH}^{n}(X)$ is the Chow group of codimension $n$ cycles. We consider the étale motivic or Lichtenbaum cohomology of $X$, i.e., the hypercohomology groups of $z^{n}(-, \bullet)$ viewed as a complex of étale sheaves,

$$
\mathrm{H}_{\mathrm{L}}^{m}(X, A(n))=\mathbb{H}_{\text {ét }}^{m}\left(X,\left(z_{n}(-, \bullet)_{\text {ét }} \otimes A\right)[-2 n]\right) ;
$$

we also set $\mathrm{H}_{\mathrm{L}}^{2 n}(X, \mathbb{Z}(n))=\mathrm{CH}_{\mathrm{L}}^{n}(X)$. It is known that the comparison map

$$
\mathrm{H}_{\mathrm{M}}^{m}(X, A(n)) \rightarrow \mathrm{H}_{\mathrm{L}}^{m}(X, A(n))
$$

is an isomorphism with rational coefficients; however, with integral coefficients this map is neither injective nor surjective in general.

We show that many of the integral properties that are known to fail for motivic cohomology groups with $\mathbb{Z}$-coefficients in fact hold for the corresponding Lichtenbaum 
groups. In particular, we formulate integral versions of the Hodge and Tate conjectures for the Lichtenbaum Chow groups which are equivalent to the usual rational statements for the classical Chow groups in many cases.

For example, if $k=\mathbb{C}$ and $X$ is a smooth projective variety, we write $\operatorname{HC}^{n}(X)_{\mathbb{Q}}$ for the usual rational Hodge conjecture [20] for $X$ in codimension $n$, i.e., the assertion that the image of the cycle map to singular cohomology

$$
c_{\mathbb{Q}}^{n}: \mathrm{CH}^{n}(X) \otimes \mathbb{Q} \rightarrow \mathrm{H}_{\mathrm{B}}^{2 n}(X, \mathbb{Q}(n))
$$

is the group of Hodge cycles $\operatorname{Hdg}^{2 n}(X, \mathbb{Q})=\mathrm{H}_{\mathrm{B}}^{2 n}(X, \mathbb{Q}(n)) \cap \mathrm{F}^{n} \mathrm{H}_{\mathrm{B}}^{2 n}(X, \mathbb{C})$. The analogous integral statement is known to be false in codimensions $n \geqslant 2$ : Atiyah and Hirzebruch [2, Theorem 6.5] have given examples of torsion cohomology classes which are not in the image of the integral cycle map, i.e., are not algebraic, and Kollar [27, pp. 134-135] has given examples of non-torsion cohomology classes which are not algebraic but have the property that some suitable multiple is algebraic. For related work, we refer to [50], [43], [53], and [54], for instance.

Since the integral Lichtenbaum groups do not admit a geometric interpretation in terms of cycles on the given variety, the usual methods of defining cycle maps via the fundamental class of a cycle and cohomology with supports cannot be applied. However, there are formal ways to prove the existence of such integral cycle maps. We show that there is an integral cycle map

$$
c_{\mathrm{L}}^{m, n}: \mathrm{H}_{\mathrm{L}}^{m}(X, \mathbb{Z}(n)) \rightarrow \mathrm{H}_{\mathrm{B}}^{m}(X, \mathbb{Z}(n))
$$

which extends the usual cycle map, and has the property that its image $\mathrm{I}_{\mathrm{L}}^{2 n}(X):=$ $\operatorname{im}\left(c_{\mathrm{L}}^{2 n, n}\right)$ lies in the group of integral Hodge cycles $\operatorname{Hdg}^{2 n}(X, \mathbb{Z})$. This map allows us to state an L-version of the integral Hodge conjecture: $\operatorname{HC}_{\mathrm{L}}^{n}(X)_{\mathbb{Z}} \Leftrightarrow \mathrm{I}_{\mathrm{L}}^{2 n}(X)=\operatorname{Hdg}^{2 n}(X, \mathbb{Z})$. Our first result shows that the usual rational Hodge conjecture is equivalent to this integral L-Hodge conjecture.

Theorem 1.1. Let $X$ be a smooth projective complex variety. Then the following hold.

(a) $\operatorname{HC}^{n}(X)_{\mathbb{Q}} \Leftrightarrow \operatorname{HC}_{\mathrm{L}}^{n}(X)_{\mathbb{Z}}$ for $n \geqslant 0$.

(b) The map $\mathrm{CH}_{\mathrm{L}}^{n}(X)_{\text {tors }} \rightarrow \operatorname{Hdg}^{2 n}(X, \mathbb{Z})_{\text {tors }}$ is surjective for $n \geqslant 0$.

We remark that the existence of an integral cycle map $c_{\mathrm{L}}^{m, n}$ as in (1) follows directly from the fact that there is such a map with rational coefficients, as well as a cycle map from Lichtenbaum cohomology to étale cohomology with $\ell$-adic coefficients. With this cycle map, Theorem 1.1 in the special case of codimension 2 follows easily from basic properties of Lichtenbaum cohomology. A key point in the proof of the general case is the construction of an integral cycle map from Lichtenbaum cohomology to Deligne-Beilinson cohomology

$$
c_{\mathrm{L}, \mathrm{D}}^{m, n}: \mathrm{H}_{\mathrm{L}}^{m}(X, \mathbb{Z}(n)) \rightarrow \mathrm{H}_{\mathrm{D}}^{m}(X, \mathbb{Z}(n))
$$

whose composition with the natural map $\mathrm{H}_{\mathrm{D}}^{m}(X, \mathbb{Z}(n)) \rightarrow \mathrm{H}_{\mathrm{B}}^{m}(X, \mathbb{Z}(n))$ is the map $c_{\mathrm{L}}^{m, n}$. To define $c_{\mathrm{L}, \mathrm{D}}^{m, n}$, we show that elements in Lichtenbaum cohomology come from higher Chow cycles on étale coverings and use a variant of Bloch's construction [7]; see Theorems 4.2 
and 4.4. This construction applies more generally to the usual cycle maps for any quasi-projective variety. Given the cycle map (2), it follows from a formal argument that, for a smooth projective complex variety, the torsion in Lichtenbaum cohomology is isomorphic to the torsion in Deligne-Beilinson cohomology, i.e., it matches the torsion in the absolute cohomology theory, in all bidegrees $(m, n)$ with $2 n-m \geqslant 0$. The analogous statement for classical Chow groups is known to be false in general.

Theorem 1.2. Let $X$ be a smooth projective complex variety, and let

$$
\left.c_{\mathrm{L}, \mathrm{D}}^{m, n}\right|_{\text {tors }}: \mathrm{H}_{\mathrm{L}}^{m}(X, \mathbb{Z}(n))_{\text {tors }} \rightarrow \mathrm{H}_{\mathrm{D}}^{m}(X, \mathbb{Z}(n))_{\text {tors }}
$$

be the restriction of the cycle map $c_{\mathrm{L}, \mathrm{D}}^{m, n}$ to torsion subgroups.

(a) If $2 n-m \geqslant 0$, then $\left.c_{\mathrm{L}, \mathrm{D}}^{m, n}\right|_{\text {tors }}$ is an isomorphism.

(b) If $2 n-m=-1$, the following statements are equivalent:

$$
\left.c_{\mathrm{L}, \mathrm{D}}^{2 n+1, n}\right|_{\text {tors }} \text { is injective } \Leftrightarrow \mathrm{HC}_{\mathrm{L}}^{n}(X)_{\mathbb{Z}} \Leftrightarrow \mathrm{HC}^{n}(X)_{\mathbb{Q}} .
$$

In section 6 , we consider integral Lichtenbaum Chow groups and the Tate conjecture [48]. Let $k$ be a field, finitely generated over the prime field, and let $X$ be a smooth projective geometrically integral $k$-variety. If $\bar{k}$ is a separable closure of $k$, we set $\bar{X}=$ $X \times{ }_{k} \bar{k}$ and $\Gamma_{k}=\operatorname{Gal}(\bar{k} / k)$. If $\ell \neq \operatorname{char}(k)$ is a prime, we denote by $\operatorname{TC}^{n}(X)_{\mathbb{Q}_{\ell}}$ the rational Tate conjecture for $X$ in codimension $n$ at the prime $\ell$, i.e., the statement that the cycle map

$$
c_{\mathbb{Q}_{\ell}}^{n}: \mathrm{CH}^{n}(X) \otimes \mathbb{Q}_{\ell} \rightarrow \mathrm{H}_{\text {ét }}^{2 n}\left(\bar{X}, \mathbb{Q}_{\ell}(n)\right)^{\Gamma_{k}}
$$

is surjective. We write $\operatorname{TC}^{n}(\bar{X})_{\mathbb{Q}_{\ell}}$ for the variant asserting that the cycle map

$$
\bar{c}_{\mathbb{Q}_{\ell}}^{n}: \mathrm{CH}^{n}(\bar{X}) \otimes \mathbb{Q}_{\ell} \rightarrow \operatorname{Ta}^{2 n}\left(\bar{X}, \mathbb{Q}_{\ell}\right):=\lim _{\rightarrow} \mathrm{H}_{\text {ét }}^{2 n}\left(\bar{X}, \mathbb{Q}_{\ell}(n)\right)^{\Gamma_{k^{\prime}}}
$$

is surjective, where the limit is taken over all fields $k \subseteq k^{\prime} \subseteq \bar{k}$ of finite degree over $k$. Again, the integral analogues of these conjectures can be stated but are known to be false; there are examples that exhibit this failure over $\mathbb{Q}$ for $n \geqslant 1$ [38, p. 493], and over $\mathbb{F}_{q}$ for $n \geqslant 2[13$, Théorème 2.1$]$.

There is a cycle map from integral Lichtenbaum cohomology to $\ell$-adic continuous étale cohomology, whose composition with the map $\mathrm{H}_{\text {cont }}^{2 n}\left(X, \mathbb{Z}_{\ell}(n)\right) \rightarrow \mathrm{H}_{\text {ét }}^{2 n}\left(\bar{X}, \mathbb{Z}_{\ell}(n)\right)^{\Gamma_{k}}$ from the Hochschild-Serre spectral sequence induces a map

$$
c_{\mathrm{L}, \mathbb{Z}_{\ell}}^{n}: \mathrm{CH}_{\mathrm{L}}^{n}(X) \otimes \mathbb{Z}_{\ell} \rightarrow \mathrm{H}_{\text {ét }}^{2 n}\left(\bar{X}, \mathbb{Z}_{\ell}(n)\right)^{\Gamma_{k}},
$$

which allows us to state integral L-analogues $\operatorname{TC}_{\mathrm{L}}^{n}(X)_{\mathbb{Z}_{\ell}}$ and $\operatorname{TC}_{\mathrm{L}}^{n}(\bar{X})_{\mathbb{Z}_{\ell}}$ of the above conjectures; for details, see $\S 6$.

For a subfield $k \subseteq \mathbb{C}$, comparison with the complex case yields the following.

Theorem 1.3. Let $k \subseteq \mathbb{C}$ be a field which is finitely generated over $\mathbb{Q}$, and let $X$ be a smooth projective geometrically integral $k$-variety. Then

$$
\operatorname{TC}^{n}(\bar{X})_{\mathbb{Q}_{\ell}} \Leftrightarrow \operatorname{TC}_{\mathrm{L}}^{n}(\bar{X})_{\mathbb{Z}_{\ell}} \text { for } n \geqslant 0 ;
$$

moreover, $\operatorname{Ta}^{2 n}\left(\bar{X}, \mathbb{Z}_{\ell}\right)_{\text {tors }}$ is contained in the image of $\mathrm{CH}_{\mathrm{L}}^{n}(X)_{\text {tors }} \otimes \mathbb{Z}_{\ell}$. 
If $k$ is a finite field, we can say more: Tate has shown that, if $X$ is a surface, $\operatorname{TC}^{1}(X)_{\mathbb{Q}_{\ell}}$ holds if and only if the $\ell$-primary torsion subgroup of the cohomological Brauer group $\operatorname{Br}(X)\{\ell\}$ is finite [46, Theorem 5.2]. Because of the quasi-isomorphism $\mathbb{Z}(1)_{\text {ét }} \simeq \mathbb{G}_{\mathrm{m}}[-1]$, we have $\mathrm{H}_{\mathrm{L}}^{3}(X, \mathbb{Z}(1)) \cong \operatorname{Br}(X)$, and one can view the Lichtenbaum group $\mathrm{H}_{\mathrm{L}}^{2 n+1}(X, \mathbb{Z}(n))$ as a higher $n$th Brauer group. We show that the usual rational Tate conjecture is again equivalent to its integral L-version, and that the $\ell$-primary torsion subgroup $\mathrm{H}_{\mathrm{L}}^{2 n+1}(X, \mathbb{Z}(n))\{\ell\}$ being finite is precisely the obstruction for it to hold.

Theorem 1.4. Let $k$ be a finite field, and let $X$ be a smooth projective geometrically integral $k$-variety. If $\ell \neq \operatorname{char}(k)$ is a prime number, then

$$
\operatorname{TC}^{n}(X)_{\mathbb{Q}_{\ell}} \Leftrightarrow \operatorname{TC}_{\mathrm{L}}^{n}(X)_{\mathbb{Z}_{\ell}} \Leftrightarrow \mathrm{H}_{\mathrm{L}}^{2 n+1}(X, \mathbb{Z}(n))\{\ell\}<\infty \quad \text { for } n \geqslant 0 .
$$

Furthermore, the torsion subgroup $\mathrm{H}_{\text {ét }}^{2 n}\left(\bar{X}, \mathbb{Z}_{\ell}(n)\right)_{\text {tors }}^{\Gamma_{k}}$ is L-algebraic.

For related results showing the equivalence of the rational Tate conjecture over a finite field to various finiteness conjectures in $K$-theory and motivic cohomology, see [16, 34, 49], for example. Assuming the existence of certain étale motivic complexes as conjectured by Lichtenbaum [30], and writing $\mathrm{H}_{\mathrm{L}}^{m}(X, \mathbb{Z}(n))$ for the corresponding hypercohomology groups, an equivalence of the form $\operatorname{TC}^{n}(X)_{\mathbb{Q}_{\ell}} \Leftrightarrow \mathrm{H}_{\mathrm{L}}^{2 n+1}(X, \mathbb{Z}(n))\{\ell\}<\infty$ has already been shown by Milne [34, Remark 5.4(g)].

In particular, it follows from our results that the integral L-versions of the Hodge and Tate conjectures we consider hold for all torsion classes.

The paper is organized as follows. In $\S 2$, we summarize basic definitions and properties of motivic and Lichtenbaum cohomology. In $\S 3$, we give examples which show how the integral Lichtenbaum cohomology groups differ from the usual motivic cohomology groups, and explain why the following, more technical, considerations are needed. Then, in $\S 4$, we give a geometric interpretation of elements in Lichtenbaum cohomology and use this description to construct cycle maps. In $\S \S 5$ and 6 , we consider the Hodge conjecture and the Tate conjecture, respectively.

Notation. If $A$ is an abelian group, $\ell$ is a prime, and $r \geqslant 1$ is an integer, we write $A\left[\ell^{r}\right]$ for the $\ell^{r}$-torsion and $A\{\ell\}=\cup_{r} A\left[\ell^{r}\right]$ for the $\ell$-primary-torsion subgroup of $A$. We set $A_{\text {tors }}=\cup_{\ell} A\{\ell\}$ and $A / \ell^{r}=A \otimes \mathbb{Z} / \ell^{r}$.

\section{Preliminaries}

Let $\mathrm{Sm}_{k}$ be the category of smooth separated $k$-schemes over a field $k$. We write $z^{n}(X, \bullet)$ for the cycle complex of abelian groups defined by Bloch [6] whose homology groups define the higher Chow groups

$$
\mathrm{CH}^{n}(X, m)=\mathrm{H}_{m}\left(z^{n}(X, \bullet)\right) .
$$

The cycle complex is contravariantly functorial for flat morphism between smooth $k$-schemes; thus $z^{n}(-, \bullet): U \mapsto z^{n}(U, \bullet)$ defines a complex of sheaves on the flat site over $X$, and therefore on the (small) étale, Nisnevich and Zariski site of $X$. If $\tau$ is any of these topologies and $A$ is an abelian group, we set

$$
A_{X}(n)_{\tau}=\left(z^{n}(-, \bullet)_{\tau} \otimes A\right)[-2 n] .
$$


For simplicity, we often write $A_{X}(n)=A_{X}(n)_{\text {Zar }}$; for example, $\mathbb{Z}_{X}(0)=\mathbb{Z}_{X}$ and $\mathbb{Z}_{X}(1) \sim$ $\mathcal{O}_{X}^{\times}[-1]\left[6\right.$, Corollary 6.4]. The hypercohomology of the complex $A_{X}(n)_{\tau}$ (which is unbounded on the left) is defined as the cohomology of the total product complex $\operatorname{Tot}(\Gamma(X, I))$, where $A_{X}(n)_{\tau} \rightarrow I$ is a Cartan-Eilenberg resolution; see [45, p. 121], for instance. We consider the cohomology groups

$$
\mathrm{H}_{\tau}^{m}(X, A(n))=\mathbb{H}_{\tau}^{m}\left(X, A_{X}(n)_{\tau}\right) .
$$

The complex of presheaves $U \mapsto z^{n}(U, \bullet)$ on $X$ has the Mayer-Vietoris property $[6,3.4]$ [9], and therefore satisfies Zariski descent [10], i.e., the evident maps $\mathrm{H}^{m}\left(z^{n}(U, \bullet)[-2 n]\right) \rightarrow$ $\mathbb{H}_{\text {Zar }}^{m}\left(U, \mathbb{Z}_{U}(n)\right)$ are isomorphisms. In particular,

$$
\mathrm{CH}^{n}(X, 2 n-m)=\mathrm{H}^{m}\left(z^{n}(X, \bullet)[-2 n]\right) \stackrel{\cong}{\rightarrow} \mathrm{H}_{\mathrm{Zar}}^{m}(X, \mathbb{Z}(n)) .
$$

The motivic and Lichtenbaum cohomology groups we consider are defined as

$$
\begin{aligned}
\mathrm{H}_{\mathrm{M}}^{m}(X, A(n)) & =\mathrm{H}_{\mathrm{Zar}}^{m}(X, A(n)), \\
\mathrm{H}_{\mathrm{L}}^{m}(X, A(n) & =\mathrm{H}_{\text {ét }}^{m}(X, A(n)) .
\end{aligned}
$$

Note that, since $\mathbb{Z}_{X}(1)_{\text {ét }} \sim \mathbb{G}_{m}[-1]$, we have $\mathrm{CH}^{1}(X)=\mathrm{CH}_{\mathrm{L}}^{1}(X)$.

For the complex defining Lichtenbaum cohomology, we have the following comparison results: if $\ell$ is a prime and $r \geqslant 1$ is an integer, Geisser and Levine have constructed quasi-isomorphisms $\left(\mathbb{Z} / \ell^{r} \mathbb{Z}\right)_{X}(n)_{\text {ét }} \stackrel{\sim}{\rightarrow} \mu_{\ell^{r}}^{\otimes n}$ if $\ell \neq \operatorname{char}(k)$ [18, Theorem 1.5] and $\left(\mathbb{Z} / \ell^{r} \mathbb{Z}\right)_{X}(n)_{\text {ét }} \stackrel{\sim}{\rightarrow} v_{r}(n)[-n]$ if $\ell=\operatorname{char}(k)\left[17\right.$, Theorem 8.5], where $v_{r}(n)$ is the $n$th logarithmic Hodge-Witt sheaf $[4,21]$. In particular, if for $n \geqslant 0$ and $\ell$ an arbitrary prime we set $\mathbb{Q} / \mathbb{Z}(n)=\oplus_{\ell} \mathbb{Q}_{\ell} / \mathbb{Z}_{\ell}(n)$, where

$$
\begin{array}{ll}
\mathbb{Q}_{\ell} / \mathbb{Z}_{\ell}(n)=\underset{\vec{r}}{\lim } \mu_{\ell^{r}}^{\otimes n}, & \ell \neq \operatorname{char}(k), \\
\mathbb{Q}_{\ell} / \mathbb{Z}_{\ell}(n)=\underset{\vec{r}}{\lim v_{r}}(n)[-n], & \ell=\operatorname{char}(k),
\end{array}
$$

it follows from the above comparisons results that there is a quasi-isomorphism

$$
(\mathbb{Q} / \mathbb{Z})_{X}(n)_{\text {ét }} \stackrel{\sim}{\rightarrow} \mathbb{Q} /\left.\mathbb{Z}(n)\right|_{X}
$$

With rational coefficients, the adjunction associated to the canonical map $\alpha: X_{\text {ét }} \rightarrow$ $X_{\text {Zar }}$ is an isomorphism; see [26, Théorème 2.6], for instance. Hence

$$
\mathbb{Q}_{X}(n) \stackrel{\cong}{\rightarrow} R \alpha_{*} \mathbb{Q}_{X}(n)_{\text {ét }}=R \alpha_{*} \alpha^{*} \mathbb{Q}_{X}(n) .
$$

If $X_{\bullet}$ is a smooth simplicial scheme, the hypercohomology of a bounded above complex $K$ of abelian $\tau$-sheaves ( $\tau=$ ét, Nis, Zar) on $\mathrm{Sm}_{k}$ is defined as

$$
\mathbb{H}_{\tau}^{m}\left(X_{\bullet}, K\right)=\operatorname{Hom}_{\mathrm{D}_{\tau}^{-}}\left(\mathbb{Z}\left(X_{\bullet}\right)_{*}, K[m]\right),
$$

where $D_{\tau}^{-}=D^{-}\left(\operatorname{AbShv}_{\tau}\left(\operatorname{Sm}_{k}\right)\right)$, and $\mathbb{Z}\left(X_{\bullet}\right)_{*}$ is the following chain complex: if $X_{i}$ is a component, let $\mathbb{Z}\left(X_{i}\right)$ be the abelian $\tau$-sheafification of the presheaf $Y \mapsto$ $\mathbb{Z}\left[\operatorname{Hom}_{\mathrm{Sm}_{k}}\left(Y, X_{i}\right)\right]$, let $\mathbb{Z}\left(X_{\bullet}\right)$ be the simplicial object obtained by applying $\mathbb{Z}(-)$ to the 
components of $X_{\bullet}$, and let $\mathbb{Z}\left(X_{\bullet}\right)_{*}$ be the associated chain complex whose differentials are the alternating sum of the maps coming from the simplicial structure [51, Appendix A]. We consider $\mathbb{Z}\left(X_{\bullet}\right)_{*}$ as a complex with $\mathbb{Z}\left(X_{i}\right)$ in degree $-i(i \geqslant 0)$. With the above definition one can use the usual formalism to set up a spectral sequence whose $E_{1}$-terms are the cohomology groups of the components of $X_{\bullet}$; however, since $\mathbb{Z}\left(X_{\bullet}\right)_{*}$ is unbounded, there are convergence issues. Also, if $X=X_{\bullet}^{0}$ is the trivial simplicial scheme, the hypercohomology of $X_{\bullet}^{0}$ defined above coincides with the usual hypercohomology of $X$. We write $s_{\geqslant-i} \mathbb{Z}\left(X_{\bullet}\right)_{*}$ for the subcomplex of $\mathbb{Z}\left(X_{\bullet}\right)_{*}$ consisting of all terms in degrees $\geqslant-i$, i.e., we set

$$
s_{\geqslant-i} \mathbb{Z}\left(X_{\bullet}\right)_{j}= \begin{cases}\mathbb{Z}\left(X_{\bullet}\right)_{j} & \text { for } j \geqslant-i \\ 0 & \text { for } j<-i\end{cases}
$$

Thus the hypercohomology of the truncated simplicial scheme $X_{\leqslant i}$ is given by

$$
\mathbb{H}_{\tau}^{m}\left(X_{\leqslant i}, K\right)=\operatorname{Hom}_{\mathrm{D}_{\tau}^{-}}\left(s_{\geqslant-i} \mathbb{Z}\left(X_{\bullet}\right)_{*}, \mathrm{~K}[m]\right) .
$$

Since the cycle complex is not contravariantly functorial for arbitrary morphisms even between smooth $k$-schemes, it does not define a presheaf on $\mathrm{Sm}_{k}$. However, if $X_{\bullet}$ is a smooth simplicial scheme with étale (thus flat) transition maps, we can define the motivic cohomology and the Lichtenbaum cohomology as the hypercohomology of the complexes $A(n)_{\text {Zar }}=\mathbb{Z}(n) \otimes A$ and $A(n)_{\text {ét }}=\mathbb{Z}(n)_{\text {ét }} \otimes A$, respectively:

$$
\begin{aligned}
& \mathrm{H}_{\mathrm{M}}^{m}\left(X_{\bullet}, A(n)\right)=\mathbb{H}_{\mathrm{Zar}}^{m}\left(X_{\bullet}, A(n)_{\mathrm{Zar}}\right)=\operatorname{Hom}_{\mathrm{D}_{\mathrm{Zar}}^{-}}\left(\mathbb{Z}\left(X_{\bullet}\right)_{*}, A(n)[m]\right), \\
& \mathrm{H}_{\mathrm{L}}^{m}\left(X_{\bullet}, A(n)\right)=\mathbb{H}_{\text {ét }}^{m}\left(X_{\bullet}, A(n)_{\text {ét }}\right)=\operatorname{Hom}_{\mathrm{D}_{\text {ét }}^{-}}\left(\mathbb{Z}\left(X_{\bullet}\right)_{*}, A(n)_{\text {ét }}[m]\right) .
\end{aligned}
$$

We remark that, using more sophisticated methods, one can define the motivic and Lichtenbaum cohomology of an arbitrary smooth simplicial scheme; see [28, Theorem 7.4.1], for example. In the following, we will apply simplicial methods only to étale hypercoverings of smooth varieties; thus the above direct definition using the complex $A(n)$ suffices for our purposes.

\section{Lichtenbaum cohomology}

We consider elementary properties of Lichtenbaum cohomology groups and give examples which show how Lichtenbaum cohomology differs from motivic cohomology. These examples motivate what follows and explain why some of the more technical considerations below are necessary.

Let $X$ be a smooth quasi-projective $k$-variety. Because of (6), it is obvious that, with rational coefficients, motivic and Lichtenbaum cohomology coincide:

$$
\mathrm{H}_{\mathrm{M}}^{m}(X, \mathbb{Q}(n)) \cong \mathrm{H}_{\mathrm{L}}^{m}(X, \mathbb{Q}(n)), \quad m \in \mathbb{Z}, \quad n \geqslant 0 .
$$

To see how these groups differ integrally, we note that the quasi-isomorphism $(\mathbb{Z} / \ell)_{X}(n)_{\text {ét }} \stackrel{\sim}{\rightarrow} \mu_{\ell^{r}}^{\otimes n}$ allows us to determine, using standard arguments, the torsion and cotorsion in Lichtenbaum cohomology.

Proposition 3.1. Let $k$ be a separably closed field, and let $X$ be a smooth projective $k$-variety. Assume that $\ell$ is a prime number different from $\operatorname{char}(k)$. Then the following hold. 
(a) $\mathrm{H}_{\mathrm{L}}^{m}(X, \mathbb{Z}(n))\{\ell\} \cong \mathrm{H}_{\text {ét }}^{m-1}\left(X, \mathbb{Q}_{\ell} / \mathbb{Z}_{\ell}(n)\right.$ for $m \neq 2 n+1$; in particular, $\mathrm{CH}_{\mathrm{L}}^{n}(X)\{\ell\} \cong$ $\mathrm{H}_{\text {ét }}^{2 n-1}\left(X, \mathbb{Q}_{\ell} / \mathbb{Z}_{\ell}(n)\right)$ for $n \geqslant 1$.

(b) $\mathrm{H}_{\mathrm{L}}^{m}(X, \mathbb{Z}(n)) \otimes \mathbb{Q}_{\ell} / \mathbb{Z}_{\ell}=0$ for $m \neq 2 n$.

Proof. Since $\mathbb{Z}_{X}(n)_{\text {ét }}$ is torsion free, the sequence of complexes of étale sheaves

$$
0 \rightarrow \mathbb{Z}_{X}(n)_{\text {ét }} \stackrel{\ell^{r}}{\rightarrow} \mathbb{Z}_{X}(n)_{\text {ét }} \rightarrow\left(\mathbb{Z} / \ell^{r} \mathbb{Z}\right)_{X}(n)_{\text {ét }} \rightarrow 0
$$

is exact, and induces for every bidegree $(m, n)$ the short exact sequence

$$
0 \rightarrow \mathrm{H}_{\mathrm{L}}^{m}(X, \mathbb{Z}(n)) / \ell^{r} \rightarrow \mathrm{H}_{\mathrm{L}}^{m}\left(X, \mathbb{Z} / \ell^{r} \mathbb{Z}(n)\right) \rightarrow \mathrm{H}_{\mathrm{L}}^{m+1}(X, \mathbb{Z}(n))\left[\ell^{r}\right] \rightarrow 0 .
$$

Taking the inverse limit over the $\operatorname{maps} \mathrm{H}_{\mathrm{L}}^{m}(X, \mathbb{Z}(n)) \rightarrow \mathrm{H}_{\mathrm{L}}^{m}\left(X, \mathbb{Z} / \ell^{r}(n)\right) \cong \mathrm{H}_{\text {ét }}^{m}\left(X, \mu_{\ell^{r}}^{\otimes n}\right)$, we obtain an $\ell$-adic cycle map for Lichtenbaum cohomology

$$
\tilde{c}_{\mathrm{L}, \mathbb{Z}_{\ell}}^{m, n}: \mathrm{H}_{\mathrm{L}}^{m}(X, \mathbb{Z}(n)) \rightarrow \mathrm{H}_{\text {ét }}^{m}\left(X, \mathbb{Z}_{\ell}(n)\right),
$$

which fits into the following commutative diagram with exact rows:

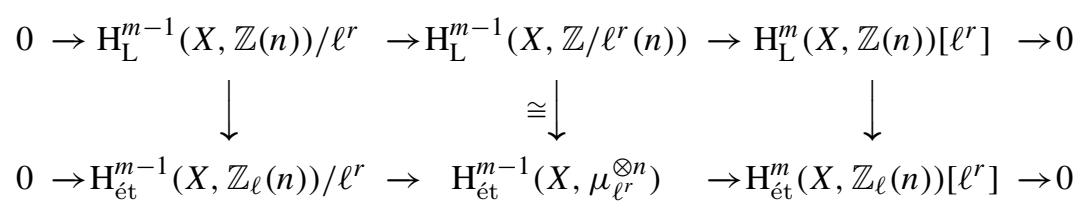

All claims follow easily from the fact that, in the diagram obtained from (10) by taking the direct limit over all powers of $\ell$, the resulting left vertical map

$$
\mathrm{H}_{\mathrm{L}}^{m-1}(X, \mathbb{Z}(n)) \otimes \mathbb{Q}_{\ell} / \mathbb{Z}_{\ell} \rightarrow \mathrm{H}_{\text {ét }}^{m-1}\left(X, \mathbb{Z}_{\ell}(n)\right) \otimes \mathbb{Q}_{\ell} / \mathbb{Z}_{\ell}
$$

is trivial if $m-1 \neq 2 n$. To see this, it suffices to show that for $m-1 \neq 2 n$ the image of (9) is contained in the finite torsion group $\mathrm{H}_{\text {ét }}^{m-1}\left(X, \mathbb{Z}_{\ell}(n)\right)_{\text {tors }}$. This follows from comparison with rational motivic cohomology, since, by a standard argument involving specialization and weights, the cycle map $\mathrm{H}_{\mathrm{M}}^{m-1}(X, \mathbb{Q}(n)) \rightarrow \mathrm{H}_{\text {ét }}^{m-1}\left(X, \mathbb{Q}_{\ell}(n)\right)$ is trivial if $m-1 \neq 2 n$; see [5, Lemma 2.4], for example.

Remarks 3.2. (a) If $k=\mathbb{C}$, we may identify étale cohomology and singular cohomology to obtain from (10) for every bidegree $(m, n)$ a surjective map on torsion groups

$$
\mathrm{H}_{\mathrm{L}}^{m}(X, \mathbb{Z}(n))_{\text {tors }} \rightarrow \mathrm{H}_{\mathrm{B}}^{m}(X, \mathbb{Z}(n))_{\text {tors }}
$$

Since for $m \geqslant 2$ there are examples of smooth projective complex varieties $X$ with the property that there are torsion classes in $\mathrm{H}_{\mathrm{B}}^{2 m}(X, \mathbb{Z}(m))$ which are not algebraic in the usual sense [2], the Lichtenbaum Chow groups $\mathrm{CH}_{\mathrm{L}}^{m}(X)$ must be 'larger' than the classical Chow groups $\mathrm{CH}^{m}(X)$, and cannot admit a description in terms of integral cycles on the given variety $X$.

(b) If $X$ is a smooth projective $k$-variety over an algebraically closed field $k$ of characteristic 0, it follows from Proposition 3.1(a) that we have an isomorphism

$$
\mathrm{CH}_{\mathrm{L}}^{2}(X)_{\text {tors }} \cong \mathrm{H}_{\text {ét }}^{3}(X, \mathbb{Q} / \mathbb{Z}(2)) \text {. }
$$


On the other hand, for the classical Chow group in codimension 2 we know that

$$
\mathrm{CH}^{2}(X)_{\text {tors }} \cong \mathrm{N}^{1} \mathrm{H}_{\text {ét }}^{3}(X, \mathbb{Q} / \mathbb{Z}(2)),
$$

where $\mathrm{N}^{1}$ stands for the first level of the coniveau filtration [12, p. 775]; hence the torsion in $\mathrm{CH}_{\mathrm{L}}^{2}(X)$ is a priori larger. When $k=\mathbb{C}$ and $\mathrm{H}^{3}\left(X, \mathcal{O}_{X}\right)=0$, the generalized Hodge conjecture [19], together with a conjecture by Nori [35], implies that $\mathrm{N}^{1} \mathrm{H}_{\text {ét }}^{3}(X, \mathbb{Q} / \mathbb{Z}(2))$ has finite index in $\mathrm{H}_{\text {ét }}^{3}(X, \mathbb{Q} / \mathbb{Z}(2))$; see $[14, \S 4]$.

(c) Under the assumptions from (b), the groups $\mathrm{H}_{\text {ét }}^{m}\left(X, \mu_{\ell}^{\otimes n}\right)$ are finite, and (10) implies in particular that $\mathrm{CH}_{\mathrm{L}}^{n}(X) / \ell$ and $\mathrm{CH}_{\mathrm{L}}^{n}(X)[\ell]$ are finite for all $n \geqslant 0$. This fails for the classical Chow groups: the examples constructed in [36, 39, 41], together with [40], show that for $d \geqslant 3$ there are examples of smooth projective $k$-varieties $X$ of dimension $d$ and primes $\ell$ such that $\# \mathrm{CH}^{p}(X) / \ell=\infty$ for $2 \leqslant p \leqslant d-1(k=\overline{\mathbb{Q}}$ or $k=\mathbb{C})$, and $\# \mathrm{CH}^{p}(X)[\ell]=\infty$ for $3 \leqslant p \leqslant d-1$ ( $k$ with positive transcendence degree or $\left.k=\mathbb{C}\right)$. We note that the known elements in these infinite cotorsion and torsion groups come from non-divisible elements of the Griffiths group $\operatorname{Griff}^{2}(X)$, which is a countable group.

(d) If $X$ is a smooth projective complex variety, its Deligne-Beilinson cohomology groups coincide with the hypercohomology groups of the complex $\mathbb{Z}_{\mathrm{D}}(n)$ of analytic sheaves $0 \rightarrow \mathbb{Z}(n) \rightarrow \mathcal{O}_{X_{\mathrm{an}}} \rightarrow \Omega_{X_{\mathrm{an}}}^{1} \rightarrow \cdots \rightarrow \Omega_{X_{\mathrm{an}}}^{n-1}$. Let $\mathbb{Q}_{\mathrm{D}}(n)=\mathbb{Z}_{\mathrm{D}}(n) \otimes \mathbb{Q}$. Then $0 \rightarrow \mathbb{Z}_{\mathrm{D}}(n) \rightarrow \mathbb{Q}_{\mathrm{D}}(n) \rightarrow \mathbb{Q} / \mathbb{Z}(n) \rightarrow 0$ is exact, and it induces a surjective map $\mathrm{H}_{\mathrm{B}}^{m-1}(X, \mathbb{Q} / \mathbb{Z}(n)) \rightarrow \mathrm{H}_{\mathrm{D}}^{m}(X, \mathbb{Z}(n))_{\text {tors }}$. The composition of the above map with the isomorphism from Proposition 3.1(a)

$$
\mathrm{H}_{\mathrm{L}}^{m}(X, \mathbb{Z}(n))_{\text {tors }} \cong \mathrm{H}_{\text {ét }}^{m-1}(X, \mathbb{Q} / \mathbb{Z}(n)) \cong \mathrm{H}_{\mathrm{B}}^{m-1}(X, \mathbb{Q} / \mathbb{Z}(n))
$$

defines for $m \neq 2 n+1$ a surjective map on torsion subgroups

$$
\mathrm{H}_{\mathrm{L}}^{m}(X, \mathbb{Z}(n))_{\text {tors }} \rightarrow \mathrm{H}_{\mathrm{D}}^{m}(X, \mathbb{Z}(n))_{\text {tors }} .
$$

Note that, if $\ell^{r}$ is a prime power, the quasi-isomorphism $\mathbb{Z}_{\mathrm{D}}(n) \otimes \mathbb{Z} / \ell^{r} \cong \mathbb{Z} / \ell^{r}(n)$ induces in every bidegree $(m, n)$ a short exact sequence of the form

$$
0 \rightarrow \mathrm{H}_{\mathrm{D}}^{m-1}(X, \mathbb{Z}(n)) / \ell^{r} \rightarrow \mathrm{H}_{\mathrm{B}}^{m-1}\left(X, \mathbb{Z} / \ell^{r}(n)\right) \rightarrow \mathrm{H}_{\mathrm{D}}^{m}(X, \mathbb{Z}(n))\left[\ell^{r}\right] \rightarrow 0 .
$$

We remark that one can describe the Lichtenbaum Chow group $\mathrm{CH}_{\mathrm{L}}^{2}(X)$ as follows: assume for simplicity that $\operatorname{char}(k)=0$. Then one has an exact sequence

$$
0 \rightarrow \mathrm{CH}^{2}(X) \rightarrow \mathrm{CH}_{\mathrm{L}}^{2}(X) \rightarrow \mathrm{H}_{\mathrm{nr}}^{3}(X, \mathbb{Q} / \mathbb{Z}(2)) \rightarrow 0,
$$

where $\mathcal{H}_{\text {ét }}^{3}(\mathbb{Q} / \mathbb{Z}(2))$ is the Zariski sheaf associated with $U \mapsto \mathrm{H}_{\text {ét }}^{3}(U, \mathbb{Q} / \mathbb{Z}(2))$, and $\mathrm{H}_{\mathrm{nr}}^{3}(X, \mathbb{Q} / \mathbb{Z}(2))=\mathrm{H}^{0}\left(X, \mathcal{H}_{\text {ét }}^{3}(\mathbb{Q} / \mathbb{Z}(2))\right)$ denotes unramified cohomology; see [26, Proposition 2.9], and also the comments below.

Remarks 3.3. (a) If $\alpha: X_{\text {ét }} \rightarrow X_{\text {Zar }}$ is the evident map, one can show that for $q \geqslant 0$ the sheaves $R^{q} \alpha_{*}\left(\mathbb{Z}_{X}(2)\right)_{\text {ét }}$ coincide with the sheaves $R^{q} \alpha_{*} \Gamma(2)$ associated with the complex $\Gamma$ (2) defined by Lichtenbaum in [30], and an exact sequence very similar to (13) already appears in [31, Theorem 2.13] (see also [24, Theorem 1.1]); we omit the details. Kahn has shown that one has an exact sequence of the form (13) for every smooth variety 
over any field [26, Proposition 2.9]; over finite fields, this sequence has been used by Colliot-Thélène and Kahn to study the unramified cohomology group $\mathrm{H}_{\mathrm{nr}}^{3}(X, \mathbb{Q} / \mathbb{Z}(2))$ [11]. We do not know a proof which shows that (13) coincides with Lichtenbaum's exact sequence; however, this seems likely to be true.

(b) By the Bloch-Ogus theory, the unramified cohomology group $\mathrm{H}_{\mathrm{nr}}^{3}(X, \mathbb{Q} / \mathbb{Z}(2))$ is closely related to the Griffiths group $\operatorname{Griff}^{2}(X) \otimes \mathbb{Q} / \mathbb{Z}[3$, Corollary 7.4 and (8.2)]; the examples constructed in $[36,39,41]$ show that, for a smooth projective variety over an algebraically closed field, the group $\operatorname{Griff}^{2}(X) \otimes \mathbb{Q} / \mathbb{Z}$ can have infinite corank. Since $\mathrm{H}_{\mathrm{nr}}^{3}(X, \mathbb{Q} / \mathbb{Z}(2))$ maps onto $\operatorname{Griff}^{2}(X) \otimes \mathbb{Q} / \mathbb{Z}$, it has infinite corank as well, and $\mathrm{CH}^{2}(X) \rightarrow$ $\mathrm{CH}_{\mathrm{L}}^{2}(X)$ is not surjective in general; however, the cokernel $\mathrm{H}_{\mathrm{nr}}^{3}(X, \mathbb{Q} / \mathbb{Z}(2))$ is again a countable group.

\section{Cycle maps}

We give a geometric interpretation of Lichtenbaum cohomology, and use it to show that the motivic cycle maps defined by Bloch in [7] extend to cycle maps on Lichtenbaum cohomology, provided the given cohomology theory satisfies étale descent. We prove these results only in characteristic 0 , which suffices for our applications; we expect them to hold more generally.

Our first lemma shows that Lichtenbaum cohomology satisfies étale descent.

Lemma 4.1. Let $X$ be a smooth $k$-scheme over a field $k$, and let $\pi: X_{\bullet} \rightarrow X$ be an étale hypercovering. Then $\mathbb{Z}\left(X_{\bullet}\right)_{*} \rightarrow \mathbb{Z}(X)$ is a quasi-isomorphism in $\mathrm{D}_{\text {ét }}^{-}=$ $\mathrm{D}^{-}\left(\mathrm{Ab} \mathrm{Shvét}_{\text {é }}\left(\mathrm{Sm}_{k}\right)\right)$. Thus for any complex of sheaves $K$ we have

$$
\mathbb{H}_{\text {èt }}^{m}(X, K)=\operatorname{Hom}_{\mathrm{D}_{\text {ét }}^{-}}(\mathbb{Z}(X), K[m]) \stackrel{\cong}{\rightrightarrows} \operatorname{Hom}_{\mathrm{D}_{\text {ét }}^{-}}\left(\mathbb{Z}\left(X_{\bullet}\right), K[m]\right)=\mathbb{H}_{\text {ét }}^{m}(X \bullet, K) .
$$

Proof. It suffices to show this with $\mathbb{Z}\left(X_{\bullet}\right)_{*}$ considered as a complex in the category $\mathrm{D}^{-}\left(\operatorname{AbShv}_{\text {ét }}\left(\operatorname{Sm}_{X}\right)\right)$, where $\operatorname{Sm}_{X}$ is the category of smooth schemes over $X$. Then $\mathbb{Z}(X)=$ $\mathbb{Z}_{X}$ is the constant sheaf $\mathbb{Z}$ on $X$, and it follows from [1, Proposition 8.5] that $\mathbb{Z}\left(X_{\bullet}\right)_{*} \rightarrow$ $\mathbb{Z}(X)=\mathbb{Z}_{X}$ is a resolution.

We will use the norm-residue isomorphism, i.e., the fact that, for every field $F$ and every prime $\ell \neq \operatorname{char}(F)$, the norm-residue map defined by Tate [47]

$$
h=h_{F}: \mathrm{K}_{m}^{M}(F) / \ell^{r} \rightarrow \mathrm{H}_{\text {ét }}^{m}\left(F, \mu_{\ell^{r}}^{\otimes m}\right)
$$

from Milnor $K$-theory to étale cohomology is an isomorphism for $m \geqslant 0$. This has been shown for special cases by Merkujev and Suslin [32, 33], and Voevodsky [51]. The general case has been established by Rost and Voevodsky [52]; see also [56]. The norm-residue isomorphism ('Bloch-Kato conjecture') is known to be equivalent to the Beilinson-Lichtenbaum conjecture [45, p. 117]. In particular, if $X$ is a smooth quasi-projective $k$-variety over a field of characteristic 0 and $\alpha: X_{\text {ét }} \rightarrow X_{\text {Zar }}$ is the canonical map, it implies that the map $\left(\mathbb{Z} / \ell^{r}\right)_{X}(n)_{\mathrm{Zar}} \rightarrow \tau_{\leqslant n} R \alpha_{*}\left(\left.\mu_{\ell^{r}}^{\otimes n}\right|_{X}\right)$ is a quasi-isomorphism for every $\ell^{r}$; hence

$$
\mathrm{H}_{\mathrm{M}}^{m}(X, \mathbb{Q} / \mathbb{Z}(n)) \cong \mathrm{H}_{\mathrm{et}}^{m}(X, \mathbb{Q} / \mathbb{Z}(n)) \quad \text { for } m \leqslant n .
$$


We show next that elements in Lichtenbaum cohomology groups come from higher Chow cycles on étale coverings of $X$. This result is an analogue of the Artin-Verdier hypercovering theorem; the proof is similar to the one for a single étale sheaf given by Artin and Mazur in [1].

Theorem 4.2. Let $k$ be a field of characteristic 0 , and let $X$ be a smooth quasi-projective $k$-variety. Then the change of topology map induces an isomorphism

$$
\underset{X_{\bullet}}{\lim _{\vec{M}}} \mathrm{H}_{\mathrm{M}}^{m}\left(X_{\bullet}, \mathbb{Z}(n)\right) \cong \mathrm{H}_{\mathrm{L}}^{m}(X, \mathbb{Z}(n)), \quad m \in \mathbb{Z}, \quad n \geqslant 0,
$$

where the limit is taken over all étale hypercoverings $X_{\bullet} \rightarrow X$.

Proof. Let $\pi: X_{\bullet} \rightarrow X$ be an étale hypercovering. We use Lemma 4.1 to identify the Lichtenbaum cohomology of $X \bullet$ and $X$ with any coefficients. From the evident distinguished triangles, we obtain the following commutative diagram with exact rows:

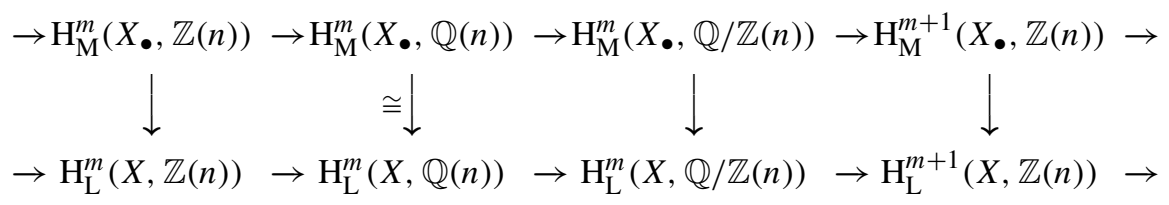

Here, the isomorphism with rational coefficients results from the fact that the adjunction $\mathbb{Q}(n) \rightarrow R \alpha_{*} \alpha^{*} \mathbb{Q}(n)$ with respect to $\alpha:\left(\mathrm{Sm}_{k}\right)_{\text {ét }} \rightarrow\left(\mathrm{Sm}_{k}\right)$ Zar is an isomorphism in $\mathrm{D}^{-}\left(\mathrm{AbShv}_{\mathrm{Zar}}\left(\mathrm{Sm}_{k}\right)\right)$. Taking the direct limit over all étale hypercoverings, it follows from the 5-lemma that it suffices to show that we have an isomorphism

$$
\underset{X_{\bullet}}{\lim _{\mathrm{M}}} \mathrm{H}_{\bullet}^{m}\left(X_{\bullet}, \mathbb{Q} / \mathbb{Z}(n)\right) \cong \mathrm{H}_{\mathrm{L}}^{m}(X, \mathbb{Q} / \mathbb{Z}(n)) .
$$

For a fixed $X_{\bullet}$, the spectral sequence which attempts to abut to the group $\mathrm{H}^{m}\left(X_{\bullet}, \mathbb{Q} / \mathbb{Z}(n)\right)$ is, as it stands, a right half-plane spectral sequence whose $\mathrm{E}_{1}^{r s}$-entries are the groups $\mathrm{H}_{\mathrm{M}}^{s}\left(X_{r}, \mathbb{Q} / \mathbb{Z}(n)\right)$. Using (14), it follows from the vanishing of étale cohomology in negative degrees that this spectral sequence is concentrated in the first quadrant, and thus we have a convergent spectral sequence:

$$
{ }_{\mathrm{M}} \mathrm{E}_{1}^{r s}=\mathrm{H}_{\mathrm{M}}^{s}\left(X_{r}, \mathbb{Q} / \mathbb{Z}(n)\right) \Rightarrow \mathrm{H}_{\mathrm{M}}^{r+s}\left(X_{\bullet}, \mathbb{Q} / \mathbb{Z}(n)\right) .
$$

On the L-side, we have an analogous spectral sequence, and we use (5) to argue similarly that $\mathrm{H}_{\mathrm{L}}^{s}\left(X_{r}, \mathbb{Q} / \mathbb{Z}(n)\right)=0$ for $s<0$. Since by Lemma $4.1 \mathrm{H}_{\mathrm{L}}^{m}(X, \mathbb{Q} / \mathbb{Z}(n)) \cong$ $\mathrm{H}_{\mathrm{L}}^{m}\left(X_{\bullet}, \mathbb{Q} / \mathbb{Z}(n)\right)$, we obtain a convergent spectral sequence:

$$
{ }_{\mathrm{L}} \mathrm{E}_{1}^{r s}=\mathrm{H}_{\mathrm{L}}^{s}\left(X_{r}, \mathbb{Q} / \mathbb{Z}(n)\right) \Rightarrow \mathrm{H}_{\mathrm{L}}^{r+s}(X, \mathbb{Q} / \mathbb{Z}(n)) .
$$

Let ${ }_{M} \mathbb{E}_{1}^{r s}$ and ${ }_{L} \mathbb{E}_{1}^{r s}$ be the $\mathrm{E}_{1}$-terms of the spectral sequences obtained from (16) and (17) by taking the limit over all étale hypercoverings $X_{\bullet} \rightarrow X$. The change of topology map induces a morphism between these spectral sequences, and to prove (15) it suffices to show that the $\mathrm{E}_{1}$-terms are isomorphic, i.e.,

$$
\mathrm{M} \mathbb{E}_{1}^{r, s} \stackrel{\cong}{\rightarrow}_{\mathrm{L}} \mathbb{E}_{1}^{r, s} \quad \text { for all }(r, s)
$$


In the L-setting, we use again (5) to identify all terms occurring in ${ }_{\mathrm{L}} \mathbb{E}_{1}^{r, s}$ with the corresponding étale cohomology groups, i.e., the cohomology groups of a single étale sheaf. Thus we can refer to [1, Proof of Theorem 8.16] to conclude that

$$
\mathrm{L}_{1}^{r s}=0 \text { if } s \neq 0 \text {. }
$$

Since the proof for the motivic case will be similar, we recall the argument. Assume that $X_{\bullet}$ is a split hypercovering of $X$ and that $\alpha \in \mathrm{H}_{\text {ét }}^{s}\left(X_{r}, \mathbb{Q} / \mathbb{Z}(n)\right)$ is a class. Write $X_{r}=\bigsqcup N_{t}$, hence $\alpha$ is the product of classes $\alpha_{t} \in \mathrm{H}_{\text {ét }}^{s}\left(N_{t}, \mathbb{Q} / \mathbb{Z}(n)\right)$. Since cohomology vanishes locally, there are coverings $N_{t}^{\prime}$ of $N_{t}$ such that $\alpha_{t} \mapsto 0$ in $\mathrm{H}_{\text {ét }}^{s}\left(N_{t}^{\prime}, \mathbb{Q} / \mathbb{Z}(n)\right)$. Moreover, one can find a refinement $X_{\bullet}^{\prime}$ of $X_{\bullet}$ such that $\alpha \mapsto 0$ in $\mathrm{H}_{\text {ét }}^{s}\left(X_{r}^{\prime}, \mathbb{Q} / \mathbb{Z}(n)\right)$. Hence $\mathbb{L}_{1}^{r, s}=0$ for $s \neq 0$. In particular, the argument only uses that cohomology classes are locally trivial and that étale hypercoverings admit suitable refinements. It follows from [44, Theorem 4.4] and $\left[42\right.$, Theorem 4.2] that for a strict Hensel local ring $S$ we have $\mathrm{H}_{\mathrm{M}}^{s}(S, \mathbb{Q} / \mathbb{Z}(n))=0$ for $s \neq 0$; thus the motivic cohomology classes are locally trivial as well, and we can argue similarly to get $\mathrm{M}_{1}^{r, s}=0$ for $s \neq 0$. The remaining non-trivial terms occur for $s=0$, in which case $\mathrm{M}_{1}^{r, 0} \cong_{\mathrm{L}} \mathbb{E}_{1}^{r, 0}$ by (14). This shows (15) and completes the proof of the theorem.

Remark 4.3. In the proof of Theorem 4.2, we use the assertion of the Beilinson-Lichtenbaum conjecture (respectively, the Bloch-Kato conjecture) to conclude that the groups $\mathrm{H}_{\mathrm{M}}^{s}\left(X_{r}, \mathbb{Q} / \mathbb{Z}(n)\right.$ vanish in negative degrees; thus the spectral sequence

$$
\mathrm{E}_{1}^{r s}=\mathrm{H}_{\mathrm{M}}^{s}\left(X_{r}, \mathbb{Q} / \mathbb{Z}(n)\right) \Rightarrow \mathrm{H}_{\mathrm{M}}^{r+s}\left(X_{\bullet}, \mathbb{Q} / \mathbb{Z}(n)\right)
$$

converges. Ayoub has informed us that one can prove Theorem 4.2 without the use of the Beilinson-Lichtenbaum conjecture, provided that one assumes that $k$ has finite cohomological dimension.

Let $X$ be a smooth quasi-projective variety over a field $k$. In [7], Bloch has shown that, if $\mathrm{H}^{m}(X, K(n))=\mathbb{H}_{\tau}^{m}\left(X, K_{X}(n)\right)$ is a cohomology theory given by a complex of sheaves $K(n)$ on $\mathrm{Sm}_{k}$ (in a suitable Grothendieck topology) which satisfies certain general properties (for example, localization sequence, homotopy invariance, weak purity, cycle map), then there are motivic cycle maps:

$$
c_{\mathrm{M}, \mathrm{K}}^{m, n}: \mathrm{H}_{\mathrm{M}}^{m}(X, \mathbb{Z}(n)) \rightarrow \mathrm{H}^{m}(X, \mathrm{~K}(n)) .
$$

We use Theorem 4.2 to show that, if such a cohomology theory also satisfies étale descent, these motivic cycle maps extend to cycle maps on Lichtenbaum cohomology. In particular, if $k=\mathbb{C}$, we obtain in this way a cycle map from Lichtenbaum to Deligne-Beilinson cohomology [15].

Theorem 4.4. Let $X$ be a smooth quasi-projective variety over a field $k$ of characteristic 0 . Assume that $\mathrm{H}^{m}(X, K(n))$ is a cohomology theory as above, which also satisfies étale decent.

(a) There is a cycle map $c_{\mathrm{L}, \mathrm{K}}^{m, n}: \mathrm{H}_{\mathrm{L}}^{m}(X, \mathbb{Z}(n)) \rightarrow \mathrm{H}^{m}(X, K(n))$, which is functorial and extends the usual motivic cycle map $c_{\mathrm{M}, \mathrm{K}}^{m, n}$. 
(b) If $\mathrm{H}^{m}(X, K(n))=\mathrm{H}_{\text {ét }}^{m}\left(X, \mu_{\ell^{r}}^{\otimes n}\right)$, the cycle map induces an isomorphism

$$
\mathrm{H}_{\mathrm{L}}^{m}\left(X, \mathbb{Z} / \ell^{r}(n)\right) \cong \mathrm{H}_{\mathrm{ét}}^{m}\left(X, \mu_{\ell^{r}}^{\otimes n}\right), \quad m \in \mathbb{Z}, \quad n \geqslant 0 .
$$

Proof. We use the notation and definitions from $\S 2$ for the hypercohomology of a smooth simplicial scheme $X_{\bullet}$ with étale transition maps and its truncations $X_{\leqslant i}$. From the inclusions $s_{\geqslant-i} \mathbb{Z}\left(X_{\bullet}\right)_{*} \rightarrow \mathbb{Z}\left(X_{\bullet}\right)_{*}$, we have maps

$$
\mathrm{H}_{\mathrm{M}}^{m}\left(X_{\bullet}, \mathbb{Z}(n)\right) \rightarrow \mathrm{H}_{\mathrm{M}}^{m}\left(X_{\leqslant i}, \mathbb{Z}(n)\right) \quad \text { and } \quad \mathrm{H}^{m}\left(X_{\bullet}, K(n)\right) \rightarrow \mathrm{H}^{m}\left(X_{\leqslant i}, K(n)\right) .
$$

We assume that for each component $X_{i}$ of $X_{\bullet}$ the groups $\mathrm{H}^{s}\left(X_{i}, K(n)\right)$ vanish in negative degrees. Thus, by the usual spectral sequence argument, the second map is an isomorphism for $i$ sufficiently large. Since the given cohomology theory satisfies étale descent, we have, for large $i$ isomorphisms,

$$
\mathrm{H}^{m}(X, K(n)) \stackrel{\cong}{\rightarrow} \mathrm{H}^{m}\left(X_{\bullet}, K(n)\right) \stackrel{\cong}{\rightarrow} \mathrm{H}^{m}\left(X_{\leqslant i}, K(n)\right) .
$$

Thus it suffices to construct for each truncation $X_{\leqslant i}$ an appropriate map:

$$
\mathrm{H}_{\mathrm{M}}^{m}\left(X_{\leqslant i}, \mathbb{Z}(n)\right) \rightarrow \mathrm{H}^{m}\left(X_{\leqslant i}, K(n)\right) .
$$

Given such maps, we can choose a sufficiently large $i$ such that we have

$$
\mathrm{H}_{\mathrm{M}}^{m}\left(X_{\bullet}, \mathbb{Z}(n)\right) \rightarrow \mathrm{H}_{\mathrm{M}}^{m}\left(X_{\leqslant i}, K(n)\right) \rightarrow \mathrm{H}^{m}\left(X_{\leqslant i}, K(n)\right) \cong \mathrm{H}^{m}(X, K(n)),
$$

and use Theorem 4.2 to define $c_{\mathrm{L}, \mathrm{K}}^{m, n}$ as the map obtained by taking the limit

$$
\mathrm{H}_{\mathrm{L}}^{m}(X, \mathbb{Z}(n)) \stackrel{\cong}{\lessgtr} \lim _{\rightarrow} \mathrm{H}_{\mathrm{M}}^{m}\left(X_{\bullet}, \mathbb{Z}(n)\right) \rightarrow \mathrm{H}^{m}(X, K(n)) .
$$

Note that, if $X$ is a smooth quasi-projective $k$-variety and $X_{\bullet} \rightarrow X$ is an étale hypercovering, there exists an étale refinement $X_{\bullet}^{\prime} \rightarrow X_{\bullet}$ with affine components. Since we will eventually take the limit over all étale hypercoverings, it suffices to construct maps as in (19) for the truncations of an étale hypercovering with affine components. Assume that $X$ • is such a smooth simplicial $k$-scheme with affine components $X_{i}$, giving an étale hypercovering of $X$. For notational simplicity, we will only do the case $X_{\leqslant 1}$; the construction for an arbitrary truncation $X_{\leqslant i}$ will be similar. Recall that in this setting we have

$$
\mathrm{H}_{\mathrm{M}}^{m}\left(X_{\leqslant 1}, \mathbb{Z}(n)\right)=\operatorname{Hom}_{\mathrm{D}^{-}\left(\mathrm{AbShv}_{\mathrm{Zar}}\left(\mathrm{Sm}_{k}\right)\right)}\left(s_{\geqslant-1} \mathbb{Z}\left(X_{\bullet}\right)_{*}, \mathbb{Z}(n)\right) .
$$

If $\mathbb{Z}_{X_{i}}(n) \rightarrow I_{X_{i}}$ is a Cartan-Eilenberg resolution, this group in degree $m$ is the $m$ th cohomology of the total complex $\operatorname{Tot}\left(X_{\leqslant 1}\right)_{\mathbb{Z}(n)}$ of the morphism of complexes

$$
\operatorname{Tot} \Gamma\left(X_{0}, I_{X_{0}}\right) \rightarrow \operatorname{Tot} \Gamma\left(X_{1}, I_{X_{1}}\right) .
$$

Let $\operatorname{Tot}\left(X_{\leqslant 1}\right)_{Z}$ be the total complex obtained from the (shifted) cycle complexes computing the higher Chow groups of the components $X_{0}$ and $X_{1}$, i.e.,

$$
z^{n}\left(X_{0}, \bullet\right)[-2 n] \rightarrow z^{n}\left(X_{1}, \bullet\right)[-2 n] .
$$


Since the cycle complex has the Mayer-Vietoris property, the two complexes $\operatorname{Tot}\left(X_{\leqslant 1}\right)_{Z}$ and $\operatorname{Tot}\left(X_{\leqslant 1}\right)_{\mathbb{Z}(n)}$ are quasi-isomorphic; we will use the isomorphism

$$
\mathrm{H}^{m}\left(\operatorname{Tot}\left(X_{\leqslant 1}\right)_{Z}\right) \cong \mathrm{H}_{\mathrm{M}}^{m}\left(X_{\leqslant 1}, \mathbb{Z}(n)\right),
$$

and work with the complex of abelian groups $\operatorname{Tot}\left(X_{\leqslant 1}\right)_{Z}$.

Consider the double complex (22). We will show that a slight reformulation of Bloch's construction gives the desired map. We recall the construction: let $W$ be a smooth quasi-projective $k$-variety, and let $K_{W}(n)$ be the restriction of $K(n)$ to $W$. Replacing $K_{W}(n)$ by its Godement resolution, we may assume that the sheaves $K_{W}(n)^{j}$ are acyclic, so that (we write $\left.\Gamma\left(K_{W}(n)^{\bullet}\right)=\Gamma\left(W, K_{W}(n)^{\bullet}\right)\right)$

$$
\mathrm{H}^{m}(W, K(n))=\mathrm{H}^{m}\left(\Gamma\left(K_{W}(n)^{\bullet}\right)\right) .
$$

If $\Delta^{p}=\Delta_{k}^{p}$ is the algebraic $p$-simplex, we obtain from the cosimplicial scheme $W \rightrightarrows$ $W \times \Delta^{1} \stackrel{\rightarrow}{\rightarrow} W \times \Delta^{2} \cdots$ an unbounded second quadrant double complex

$$
\Gamma\left(K_{W \times \Delta^{-p}}(n)\right)^{q}, \quad p, q \geqslant 0,
$$

together with a spectral sequence whose $\mathrm{E}_{1}$-terms are

$$
\mathrm{E}_{1}^{p q}=\mathrm{H}^{q}\left(W \times \Delta^{-p}, K(n)\right) .
$$

Using the assumption of homotopy invariance, it follows that the $\mathrm{E}_{2}$-terms are

$$
\mathrm{E}_{2}^{p q}= \begin{cases}\mathrm{H}^{q}(W, K(n)) & p=0 \\ 0 & p \neq 0 .\end{cases}
$$

Since the $d_{1}$-differentials are either trivial or isomorphisms, we may truncate the cosimplicial scheme at any sufficiently large level $W \times \Delta^{N}$ with $N$ even without changing anything. Taking such a truncation, we obtain a double complex $\Gamma_{\geqslant-N}\left(K_{W \times \Delta} \bullet(n)^{\bullet}\right)$ which is bounded on the left. If $\operatorname{Tot}(W)_{K(n)}$ is the corresponding total complex, we have a convergent spectral sequence:

$$
\mathrm{E}_{1}^{p q}=\mathrm{H}^{q}\left(W \times \Delta^{-p}, K(n)\right) \Rightarrow \mathrm{H}^{p+q}\left(\operatorname{Tot}(W)_{K(n)}\right)=\mathrm{H}^{p+q}(W, K(n)) .
$$

Let $\Gamma_{S}\left(K_{W \times \Delta} \cdot(n)^{\bullet}\right)$ be the similarly truncated double complex

$$
\Gamma_{S}\left(K_{W \times \Delta^{-p}}(n)^{q}\right)=\lim _{\rightarrow} \Gamma_{|Z|}\left(K_{W \times \Delta^{-p}}(n)\right)^{q},
$$

where the limit is taken over all cycles $Z \in z^{n}(W, p)$ with support $|Z|$. Let $\operatorname{Tot}_{S}(W)_{K(n)}$ be the total complex of the bicomplex $\Gamma_{S}\left(K_{W \times \Delta^{\bullet}}(n)^{\bullet}\right)$. Thus, if

$$
\mathrm{H}_{S}^{m}\left(W \times \Delta^{p}, K(n)\right)=\lim _{\rightarrow} \mathrm{H}_{|Z|}^{m}\left(W \times \Delta^{p}, K(n)\right),
$$

we have again a convergent spectral sequence:

$$
{ }_{S} \mathrm{E}_{1}^{p, q}=\mathrm{H}_{S}^{q}\left(W \times \Delta^{-p}, K(n)\right) \Rightarrow \mathrm{H}^{p+q}\left(s \operatorname{Tot}(W)_{K(n)}\right),
$$


where ${ }_{S} \mathrm{E}_{1}^{p, q}=0$ for $q<2 n$ by the purity assumption on the cohomology theory. We use this to modify $\Gamma_{S}\left(K_{W \times \Delta} \bullet(n)^{\bullet}\right)$ as follows. Consider the segment

$$
\begin{array}{lll}
\cdots \rightarrow \Gamma_{S}\left(K_{W \times \Delta^{-2}}(n)\right)^{2 n+1} & \rightarrow \Gamma_{S}\left(K_{W \times \Delta^{-1}}(n)\right)^{2 n+1} & \rightarrow \Gamma_{S}\left(K_{W}(n)\right)^{2 n+1} \rightarrow 0 \\
d_{S}^{-2,2 n \uparrow} \uparrow & d_{S}^{-1,2 n \uparrow} \uparrow & d_{S}^{0,2 n \uparrow} \\
\cdots \rightarrow \Gamma_{S}\left(K_{W \times \Delta^{-2}}(n)\right)^{2 n} & \rightarrow \Gamma_{S}\left(K_{W \times \Delta^{-1}}(n)\right)^{2 n} & \rightarrow \Gamma_{S}\left(K_{W}(n)\right)^{2 n} \rightarrow 0 \\
d_{S}^{-2,2 n-1} \uparrow & d_{S}^{-1,2 n-1} \uparrow & d_{S}^{0,2 n-1} \uparrow \\
\cdots \rightarrow \Gamma_{S}\left(K_{W \times \Delta^{-2}}(n)\right)^{2 n-1} & \rightarrow \Gamma_{S}\left(K_{W \times \Delta^{-1}}(n)\right)^{2 n-1} & \rightarrow \Gamma_{S}\left(K_{W}(n)\right)^{2 n-1} \rightarrow 0
\end{array}
$$

Let $\operatorname{Tot}_{S}^{\prime}(W)_{K(n)}$ be the total complex of the double complex $\Gamma_{S}^{\prime}\left(K_{W \times \Delta} \bullet(n)^{\bullet}\right)$ obtained from $\Gamma_{S}\left(K_{W \times \Delta} \cdot(n)^{\bullet}\right)$ by truncating all rows below $2 n$, putting

$$
\cdots \rightarrow \operatorname{coker} d_{S}^{-2,2 n-1} \rightarrow \operatorname{coker} d_{S}^{-1,2 n-1} \rightarrow \operatorname{coker}_{S} d^{0,2 n-1} \rightarrow 0
$$

in row $2 n$, and keeping all other rows above. It follows from purity that the quotient map $\operatorname{Tot}_{S}(W)_{K(n)} \rightarrow \operatorname{Tot}_{S}^{\prime}(W)_{K(n)}$ is a quasi-isomorphism. Thus we have a map in the derived category $\mathrm{D}(\mathrm{Ab})$ which is determined by the diagram

$$
\operatorname{Tot}_{S}^{\prime}(W)_{K(n)} \stackrel{\sim}{\leftarrow} \operatorname{Tot}_{S}(W)_{K(n)} \stackrel{\varphi}{\rightarrow} \operatorname{Tot}(W)_{K(n)},
$$

where $\varphi$ is the map forgetting supports. By assumption, we have a cycle map $Z^{n}(W \times$ $\left.\Delta^{p}\right) \rightarrow \mathrm{H}_{S}^{2 n}\left(W \times \Delta^{p}, K(n)\right)$. The restriction to $z^{n}(W, p)$ gives us a map with values in the bottom row of $\Gamma_{S}^{\prime}\left(K_{W \times \Delta} \cdot(n)^{\bullet}\right)$, which is compatible with the differentials of the cycle complex and the horizontal differentials in the double complex. Hence we have a diagram of maps of complexes

$$
z^{n}(W, \bullet)[-2 n] \rightarrow \operatorname{Tot}_{S}^{\prime}(W)_{K(n)} \stackrel{\sim}{\leftarrow} \operatorname{Tot}_{S}(W)_{K(n)} \stackrel{\varphi}{\rightarrow} \operatorname{Tot}(W)_{K(n)}
$$

and the map $\mathrm{H}_{\mathrm{M}}^{m}(W, \mathbb{Z}(n)) \rightarrow \mathrm{H}^{m}(W, K(n))$ obtained by taking cohomology is the motivic cycle map defined by Bloch.

To define the cycle map for $X_{\leqslant 1}$, we apply the above construction to the components of $X_{\leqslant 1}$ to obtain the commutative diagram of maps of complexes:

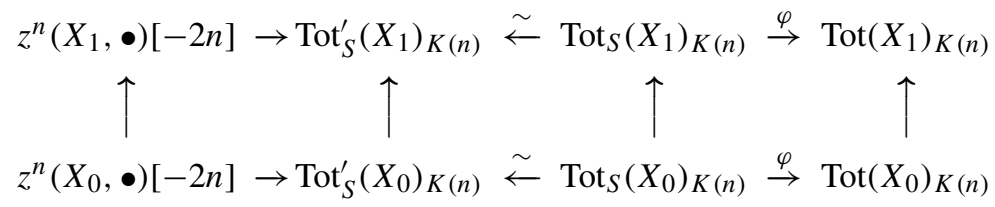

Taking total complexes and cohomology in degree $m$ defines the desired map:

$$
\mathrm{H}_{\mathrm{M}}^{m}\left(X_{\leqslant 1}, \mathbb{Z}(n)\right) \rightarrow \mathrm{H}^{m}\left(X_{\leqslant 1}, K(n)\right) .
$$

It is evident from the construction that the resulting cycle map $c_{\mathrm{L}, \mathrm{K}}^{m, n}$ extends Bloch's map $c_{\mathrm{M}, \mathrm{K}}^{m, n}$. To prove functoriality, let $f: X \rightarrow Y$ be an arbitrary morphism, and write $f_{i}: X_{i} \rightarrow Y_{i}$ for the induced morphism on the (affine) components of étale hypercoverings 
$X_{\bullet} \rightarrow X$ and $Y_{\bullet} \rightarrow Y$. It suffices to show that there are pullback maps $f_{i}^{*}$ such that the following diagram commutes:

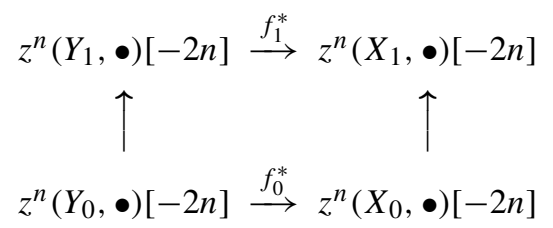

If $\mathcal{T}_{i}$ is a finite set of closed subsets of $Y_{i}$, including $Y_{i}$ as an element, define a subcomplex $z^{n}\left(Y_{i}, \bullet\right) \mathcal{T}_{i} \subseteq z^{n}\left(Y_{i}, \bullet\right)$, where $z^{n}\left(Y_{i}, p\right) \mathcal{T}_{i}$ is the subgroup generated by subvarieties which intersect $T \times F$ properly on $Y_{i} \times \Delta^{p}$ for all $T \in \mathcal{T}_{i}$ and all faces $F$ of $\Delta^{p}$. With respect to the given morphism $f_{i}: X_{i} \rightarrow Y_{i}$, set

$$
\begin{aligned}
T_{j}\left(f_{i}\right) & =\left\{y \in Y_{i} \mid \operatorname{dim}_{\kappa(y)} f_{i}^{-1}(y) \geqslant j\right\}, \\
\mathcal{T}\left(f_{i}\right) & =\left\{T_{j}\left(f_{i}\right) \mid j=-1,0,1, \ldots\right\} .
\end{aligned}
$$

Assume that $\mathcal{T}\left(f_{i}\right) \subseteq \mathcal{T}_{i}$ and that $W \in z^{n}\left(Y_{i}, p\right) \mathcal{T}_{i}$. Since $Y_{i}$ is smooth, the usual pullback $\left(f_{i} \times \mathrm{id}\right)^{*}(W)$ is defined and lies in $z^{n}\left(X_{i}, p\right)$; thus there is a pullback map:

$$
f_{i, \mathcal{T}_{i}}^{*}: z^{n}\left(Y_{i}, \bullet\right)_{\mathcal{T}_{i}} \rightarrow z^{n}\left(X_{i}, \bullet\right)
$$

Since $Y_{i}$ is also affine, it follows from a result by Bloch [8, Proposition 2.5.1] (see also [29, Part I, Chapter II, 3.5]) that the inclusion $j_{\mathcal{T}_{i}}: z^{n}\left(Y_{i}, \bullet\right)_{\mathcal{T}_{i}} \subseteq z^{n}\left(Y_{i}, \bullet\right)$ is a quasi-isomorphism. Thus we have in $\mathrm{D}(\mathrm{Ab})$ the commutative diagram

$$
\begin{aligned}
z^{n}\left(Y_{1}, \bullet\right)[-2 n] & \stackrel{\sim}{\leftarrow} z^{n}\left(Y_{1}, \bullet\right)_{\mathcal{T}_{1}}[-2 n] \stackrel{f_{1, \mathcal{T}_{1}}^{*}}{\longrightarrow} z^{n}\left(X_{1}, \bullet\right)[-2 n] \\
\uparrow & \uparrow \\
z^{n}\left(Y_{0}, \bullet\right)[-2 n] & \stackrel{\sim}{\leftarrow} z^{n}\left(Y_{0}, \bullet\right)_{\mathcal{T}_{0}}[-2 n] \stackrel{f_{0, \mathcal{T}_{0}}^{\longrightarrow}}{\longrightarrow} z^{n}\left(X_{0}, \bullet\right)[-2 n]
\end{aligned}
$$

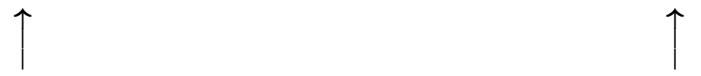

which implies (24) and completes the proof of (a).

For the second claim (b), consider again a smooth affine component $W=X_{i}$ of $X_{\bullet}$. Let now $\Gamma\left(K_{W}(n)^{\bullet}\right)$ be the complex of abelian groups obtained by taking global sections of the Godement resolution of the étale sheaf $\mu_{\ell^{r}}^{\otimes n}$ on $W$, and let $\operatorname{Tot}(W)_{K(n)}$ be the total complex associated with the double complex $\Gamma\left(K_{W \times \Delta} \bullet(n)^{\bullet}\right)$. From the above variant of Bloch's construction, we have a map

$$
c_{W}^{n}:\left(z^{n}(W, \bullet)[-2 n] \otimes \mathbb{Z} / \ell^{r} \rightarrow \operatorname{Tot}(W)_{K(n)} .\right.
$$

On the other hand, there is a subcomplex $z^{n}(W, \bullet) \mathcal{T} \subseteq z^{n}(W \bullet)$ (defined analogously to the subcomplexes $z^{n}\left(Y_{i}, \bullet\right)_{\mathcal{T}}$ considered above) with the property that $z^{n}(W, \bullet) \mathcal{T} \subseteq z^{n}(W, \bullet)$ is a quasi-isomorphism, together with a cycle map

$$
\mathrm{cl}_{W}^{n}:\left(z^{n}(W, \bullet)_{\mathcal{T}}[-2 n]\right) \otimes \mathbb{Z} / \ell^{r} \rightarrow \Gamma\left(K_{W}(n)^{\bullet}\right),
$$

which is induced from the usual cycle map [18, (2.3) and 3.7]. Thus the diagram

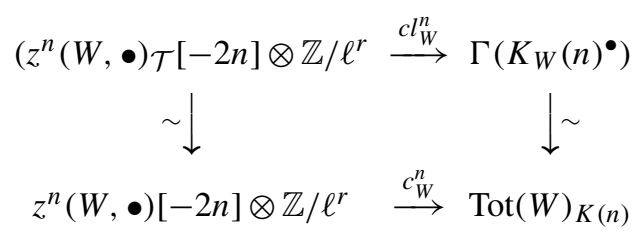


commutes, where the right quasi-isomorphism is induced from the augmentation. In particular, we may view the map on the truncated simplicial scheme

$$
\mathrm{H}_{\mathrm{M}}^{m}\left(X_{\leqslant i}, \mathbb{Z} / \ell^{r}(n)\right) \rightarrow \mathrm{H}_{\text {ét }}^{m}\left(X_{\leqslant i}, \mu_{\ell^{r}}^{\otimes n}\right)
$$

constructed above as induced from $c l_{W}^{n}$, and write $\mathrm{H}^{m}\left(c l_{W \leqslant i}^{n}\right)$ for this map. There is a sheaf version, i.e., a morphism of complexes of étale sheaves $c l_{\text {ét }}^{n}:\left(\mathbb{Z} / \ell^{r}\right)_{X}(n)_{\text {ét }} \rightarrow \mu_{\ell^{r}}^{\otimes n}$ which is the quasi-isomorphism from [18, Theorem 1.5]. Since we work with an étale hypercovering, the morphism $c l_{\text {ét }}^{n}$ induces a map

$$
\mathrm{H}^{m}\left(c l_{\text {ét } \leqslant i}^{n}\right): \mathrm{H}_{\mathrm{L}}^{m}\left(X_{\leqslant i}, \mathbb{Z} / \ell^{r}(n)\right) \rightarrow \mathrm{H}_{\text {ét }}^{m}\left(X_{\leqslant i}, \mu_{\ell^{r}}^{\otimes n}\right),
$$

which fits into the following commutative diagram:

$$
\begin{array}{cc}
\mathrm{H}_{\mathrm{M}}^{m}\left(X_{\bullet}, \mathbb{Z} / \ell^{r}(n)\right) \rightarrow & \mathrm{H}_{\mathrm{M}}^{m}\left(X_{\leqslant i}, \mathbb{Z} / \ell^{r}(n)\right) \stackrel{\mathrm{H}^{m}\left(c l_{W \leqslant i}^{n}\right)}{\longrightarrow} \mathrm{H}_{\text {ét }}^{m}\left(X_{\leqslant i}, \mu_{\ell^{r}}^{\otimes n}\right) \\
\downarrow & \downarrow= \\
\mathrm{H}_{\mathrm{L}}^{m}\left(X_{\bullet}, \mathbb{Z} / \ell^{r}(n)\right) \rightarrow & \mathrm{H}_{\mathrm{L}}^{m}\left(X_{\leqslant i}, \mathbb{Z} / \ell^{r}(n)\right) \stackrel{\mathrm{H}^{m}\left(c l_{\text {êt } \leqslant i}^{n}\right)}{\longrightarrow} \mathrm{H}_{\text {ét }}^{m}\left(X_{\leqslant i}, \mu_{\ell^{r}}^{\otimes n}\right)
\end{array}
$$

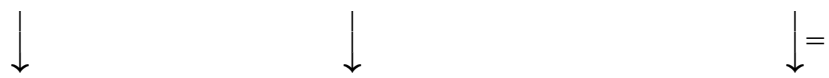

Choose $i$ large enough, so that $\mathrm{H}_{\text {ét }}^{m}\left(X_{\leqslant i}, \mu_{\ell^{r}}^{\otimes n}\right)=\mathrm{H}_{\text {ét }}^{m}\left(X, \mu_{\ell^{r}}^{\otimes n}\right)$. By étale descent $\mathrm{H}_{\mathrm{L}}^{m}(X, \mathbb{Z}(n)) \cong \mathrm{H}_{\mathrm{L}}^{m}\left(X_{\bullet}, \mathbb{Z}(n)\right)$, and the composition of the lower horizontal maps is the isomorphism induced by the quasi-isomorphism $c l_{\text {ét }}^{n}$ of Geisser and Levine. It follows from Theorem 4.2 that, after taking the limit of the above diagram over all étale hypercoverings of $X$, the resulting left vertical map is an isomorphism. Thus the composition of the upper horizontal maps is an isomorphism, which is our cycle map.

Remark 4.5. The referee has pointed out the following alternate approach to the construction of the cycle maps: the complexes with support and their canonical truncations are complexes with cohomology supported in one degree $2 n$. One has the usual $t$-structure on $\mathrm{D}(\mathrm{Ab})$ with heart $\mathrm{Ab}$, and the full subcategory of complexes with cohomology supported in degree $2 n$ is equivalent to $\mathrm{Ab}$; via this equivalence, the cycle map to cohomology with supports is immediately seen to be a functorial map of complexes, giving the wanted cycle map.

\section{Hodge conjecture}

Let $X$ be a smooth projective complex variety. We use the cycle map from Lichtenbaum to Deligne-Beilinson cohomology from Theorem 4.4 to prove Theorem 1.1. Since the codimension 2 case can be done by bare hands, we begin with this special case.

Note first that, because of the $\ell$-adic cycle map (9) and the rational comparison isomorphism between motivic and Lichtenbaum groups, there is a unique integral cycle map $c_{\mathrm{L}}^{m, n}$ as in (1); the map $c_{\mathrm{L}}^{m}=c_{\mathrm{L}}^{2 m, m}$ is a special case. More precisely, using the isomorphism (7), we have with rational coefficients a map

$$
c_{\mathrm{L}, \mathbb{Q}}^{m, n}: \mathrm{H}_{\mathrm{L}}^{m}(X, \mathbb{Q}(n)) \rightarrow \mathrm{H}_{\mathrm{B}}^{m}(X, \mathbb{Q}(n))
$$


From the evident Cartesian square

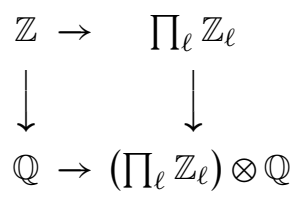

we obtain the exact sequence

$$
0 \rightarrow \mathbb{Z} \rightarrow \mathbb{Q} \oplus \prod_{\ell} \mathbb{Z}_{\ell} \rightarrow\left(\prod_{\ell} \mathbb{Z}_{\ell}\right) \otimes \mathbb{Q} \rightarrow 0,
$$

which is universally exact, since $\mathbb{Z}_{\ell} \otimes \mathbb{Q}$ is torsion free. If $\iota: \mathbb{Z} \rightarrow \mathbb{Q}$ is the inclusion, and $\iota_{\ell}$ denotes the composition $\mathbb{Z}_{\ell} \rightarrow \mathbb{Z}_{\ell} \otimes \mathbb{Q} \rightarrow \mathbb{Q}_{\ell}$, the sequence

$$
0 \rightarrow \mathrm{H}_{\mathrm{B}}^{m}(X, \mathbb{Z}(n)) \rightarrow \mathrm{H}_{\mathrm{B}}^{m}(X, \mathbb{Q}(n)) \oplus \prod_{\ell} \mathrm{H}_{\text {ét }}^{m}\left(X, \mathbb{Z}_{\ell}(n)\right) \stackrel{\iota-\iota \ell}{\rightarrow} \prod_{\ell} \mathrm{H}_{\text {ét }}^{m}\left(X, \mathbb{Q}_{\ell}(n)\right)
$$

is exact; here, we have used that $\mathrm{H}_{\mathrm{B}}^{m}(X, \mathbb{Z}(n))$ is a finitely generated abelian group. For $z \in \mathrm{H}_{\mathrm{L}}^{m}(X, \mathbb{Z}(n))$, the images of its classes $c_{\mathrm{L}, \mathbb{Q}}^{m, n}(z)$ and $\tilde{c}_{\mathrm{L}, \mathbb{Z}_{\ell}}^{m, n}(z)$ under the maps $\iota$ and $\iota_{\ell}$ coincide in $\prod_{\ell} \mathrm{H}_{\text {ét }}^{m}\left(X, \mathbb{Q}_{\ell}(n)\right)$ by the comparison theorems; hence there is a unique integral cycle class map $c_{\mathrm{L}}^{m, n}$, as claimed.

Let $\mathrm{I}^{2 n}(X)$ be the image of the map $c^{n}: \mathrm{CH}^{n}(X) \rightarrow \mathrm{H}_{\mathrm{B}}^{2 n}(X, \mathbb{Z}(n))$. Thus

$$
\mathrm{Z}^{2 n}(X)=\operatorname{coker}\left\{\mathrm{I}^{2 n}(X) \rightarrow \operatorname{Hdg}^{2 n}(X, \mathbb{Z})\right\}
$$

is the obstruction to the integral Hodge conjecture in codimension $n$, i.e., the integral Hodge conjecture $\mathrm{HC}^{n}(X)_{\mathbb{Z}}$ is equivalent to $\mathrm{Z}^{2 n}(X)=0$, and the rational Hodge conjecture $\operatorname{HC}^{n}(X)_{\mathbb{Q}}$ to $\mathrm{Z}^{2 n}(X)$ being a finite group. Similarly, let $\mathrm{I}_{\mathrm{L}}^{2 n}(X)$ be the image of the map $c_{\mathrm{L}}^{n}: \mathrm{CH}_{\mathrm{L}}^{n}(X) \rightarrow \mathrm{H}_{\mathrm{B}}^{2 n}(X, \mathbb{Z}(n))$, and write

$$
\mathrm{Z}_{\mathrm{L}}^{2 n}(X)=\operatorname{coker}\left\{\mathrm{I}_{\mathrm{L}}^{2 n}(X) \rightarrow \operatorname{Hdg}^{2 n}(X, \mathbb{Z})\right\}
$$

for the obstruction to the integral L-Hodge conjecture $\mathrm{HC}_{\mathrm{L}}^{n}(X)_{\mathbb{Z}}$. Because of the inclusions $\mathrm{I}^{2 n}(X) \subseteq \mathrm{I}_{\mathrm{L}}^{2 n}(X) \subseteq \operatorname{Hdg}^{2 n}(X, \mathbb{Z})$, there is an exact sequence

$$
0 \rightarrow \mathrm{I}_{\mathrm{L}}^{2 n}(X) / \mathrm{I}^{2 n}(X) \rightarrow \mathrm{Z}^{2 n}(X) \rightarrow \mathrm{Z}_{\mathrm{L}}^{2 n}(X) \rightarrow 0
$$

whose first term is finite, since $\mathrm{I}^{2 n}(X)$ and $\mathrm{I}_{\mathrm{L}}^{2 n}(X)$ have the same $\mathbb{Z}$-rank.

Assume now that $n=2$. From the compatibility of $c^{2}$ and $c_{\mathrm{L}}^{2}$, together with the exact sequence (13), we obtain the following commutative diagram with exact rows:

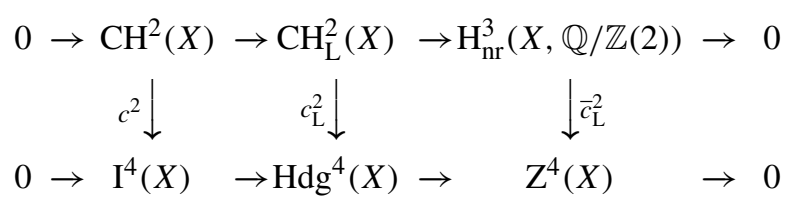

The induced map $\bar{c}_{\mathrm{L}}^{2}: \mathrm{H}_{\mathrm{nr}}^{3}(X, \mathbb{Q} / \mathbb{Z}(2)) \rightarrow \mathrm{Z}^{4}(X)_{\text {tors }}$ can be identified with the surjective map $\mathrm{H}_{\mathrm{nr}}^{3}(X, \mathbb{Q} / \mathbb{Z}(2)) \rightarrow \mathrm{H}^{1}\left(X, \mathcal{H}_{B}^{3}(\mathbb{Z}(2))\right)_{\text {tors }}$; this uses that the sheaves $\mathcal{H}_{B}^{3}(\mathbb{Z}(2))$ and $\mathcal{H}_{B}^{4}(\mathbb{Z}(2))$ are torsion free and the isomorphism

$$
\mathrm{Z}^{4}(X)_{\text {tors }} \cong\left[\mathrm{H}_{\mathrm{B}}^{4}(X, \mathbb{Z}(2)) / \mathrm{I}^{4}(X)\right]_{\text {tors }} \cong \mathrm{H}^{1}\left(X, \mathcal{H}_{B}^{3}(\mathbb{Z}(2))\right)_{\text {tors }}
$$


In particular, it follows that the obstruction to the integral L-Hodge conjecture

$$
\mathrm{Z}_{\mathrm{L}}^{4}(X)=\operatorname{coker}\left(\bar{c}_{\mathrm{L}}^{2}\right)
$$

is torsion free, and the exact sequence (27) implies the claimed equivalence:

$$
\mathrm{HC}^{2}(X)_{\mathbb{Q}} \Leftrightarrow \mathrm{Z}^{4}(X) \otimes \mathbb{Q}=0 \Leftrightarrow \mathrm{Z}_{\mathrm{L}}^{4}(X)=0 \Leftrightarrow \mathrm{HC}_{\mathrm{L}}^{2}(X)_{\mathbb{Z}} .
$$

In [14, Théorème 3.7], Colliot-Thélène and Voisin showed that the sequence

$$
0 \rightarrow \mathrm{H}_{\mathrm{nr}}^{3}(X, \mathbb{Z}(2)) \otimes \mathbb{Q} / \mathbb{Z} \rightarrow \mathrm{H}_{\mathrm{nr}}^{3}(X, \mathbb{Q} / \mathbb{Z}(2)) \rightarrow \mathrm{Z}^{4}(X)_{\text {tors }} \rightarrow 0
$$

is exact, and used it to study the integral Hodge conjecture. We remark that, using our approach, this exact sequence follows easily from the above diagram (by using (28) to identify the kernel of $\bar{c}_{\mathrm{L}}^{2}$ with $\left.\mathrm{H}_{\mathrm{nr}}^{3}(X, \mathbb{Z}(2)) \otimes \mathbb{Q} / \mathbb{Z}\right)$.

For the general case, we need to use both cycle maps (1) and (2). We will write $\mathrm{H}_{\mathrm{L}}^{m}(X, \mathbb{Z}(n))_{\text {hom }}=\operatorname{ker}\left(c_{\mathrm{L}}^{m, n}\right)$ and $\mathrm{I}_{\mathrm{L}}^{m, n}(X)=\operatorname{im}\left(c_{\mathrm{L}}^{m, n}\right)$; we also set $\mathrm{CH}_{\mathrm{L}}^{n}(X)_{\text {hom }}=$ $\mathrm{H}_{\mathrm{L}}^{2 n}(X, \mathbb{Z}(n))_{\text {hom }}$ and $\operatorname{im}\left(c_{\mathrm{L}}^{n}\right)=\mathrm{I}_{\mathrm{L}}^{2 n}(X)=\mathrm{I}_{\mathrm{L}}^{2 n, n}(X)=\operatorname{im}\left(c_{\mathrm{L}}^{2 n, n}\right)$.

Proposition 5.1. Let $X$ be a smooth projective complex variety.

(a) If $2 n-m \geqslant 0$, the restriction of $c_{\mathrm{L}, \mathrm{D}}^{m, n}$ to torsion is an isomorphism. Thus $\mathrm{H}_{\mathrm{L}}^{m}(X, \mathbb{Z}(n))_{\text {tors }} \cong \mathrm{H}_{\mathrm{D}}^{m}(X, \mathbb{Z}(n))_{\text {tors }} \cong \mathrm{H}_{\mathrm{B}}^{m-1}(X, \mathbb{Q} / \mathbb{Z}(n))$. Furthermore, the cokernel of $c_{\mathrm{L}, \mathrm{D}}^{m, n}$ is torsion free.

(b) If $n \geqslant 0$, then $\mathrm{CH}_{\mathrm{L}}^{n}(X)_{\text {hom }} \otimes \mathbb{Q} / \mathbb{Z}=0$.

(c) Let $\Phi_{X}^{n}: \mathrm{CH}^{n}(X)_{\mathrm{hom}} \rightarrow \mathrm{J}^{n}(X)$ be the Abel-Jacobi map. Then

$$
\operatorname{ker}\left(\Phi_{X}^{n}\right)_{\text {tors }}=\operatorname{ker}\left\{\mathrm{CH}^{n}(X) \rightarrow \mathrm{CH}_{\mathrm{L}}^{n}(X)\right\} \quad \text { for } n \geqslant 0 .
$$

Proof. Using (8), (12) and Theorem 4.4(b), we have a commutative diagram

$$
\begin{aligned}
& 0 \rightarrow \mathrm{H}_{\mathrm{L}}^{m-1}(X, \mathbb{Z}(n)) / \ell^{r} \rightarrow \mathrm{H}_{\mathrm{L}}^{m-1}\left(X, \mathbb{Z} / \ell^{r}(n)\right) \rightarrow \mathrm{H}_{\mathrm{L}}^{m}(X, \mathbb{Z}(n))\left[\ell^{r}\right] \rightarrow 0 \\
& \left.c_{\mathrm{L}, \mathrm{D}}^{m-1, n} / \ell^{r} \downarrow \quad \cong \downarrow c_{\mathrm{L}, \mathrm{D}}^{m, n} \mid \ell^{r}\right] \\
& 0 \rightarrow \mathrm{H}_{\mathrm{D}}^{m-1}(X, \mathbb{Z}(n)) / \ell^{r} \rightarrow \mathrm{H}_{\mathrm{B}}^{m-1}\left(X, \mathbb{Z} / \ell^{r}(n)\right) \rightarrow \mathrm{H}_{\mathrm{D}}^{m}(X, \mathbb{Z}(n))\left[\ell^{r}\right] \rightarrow 0
\end{aligned}
$$

which shows that the restriction $\left.c_{\mathrm{L}, \mathrm{D}}^{m, n}\right|_{\left[\ell^{r}\right]}$ is surjective for all $(m, n)$.

Since $X$ is projective, a standard Hodge theoretic argument implies that

$$
\mathrm{H}_{\mathrm{D}}^{m-1}(X, \mathbb{Z}(n)) \otimes \mathbb{Z} / \ell^{r} \cong \mathrm{H}_{\mathrm{B}}^{m-1}(X, \mathbb{Z}(n))_{\text {tors }} \otimes \mathbb{Z} / \ell^{r} \quad \text { if } 2 n-m+1>0 .
$$

Hence $\mathrm{H}_{\mathrm{D}}^{m-1}(X, \mathbb{Z}(n)) \otimes \mathbb{Q} / \mathbb{Z}=0$, and it follows from (29) that $\left.c_{\mathrm{L}, \mathrm{D}}^{m, n}\right|_{\text {tors }}$ is injective, and therefore an isomorphism, provided that $2 n-m \geqslant 0$. Let $A=\mathrm{H}_{\mathrm{L}}^{m}(X, \mathbb{Z}(n))$ and $B=\mathrm{H}_{\mathrm{D}}^{m}(X, \mathbb{Z}(n))$, where again $2 n-m \geqslant 0$. In the diagram

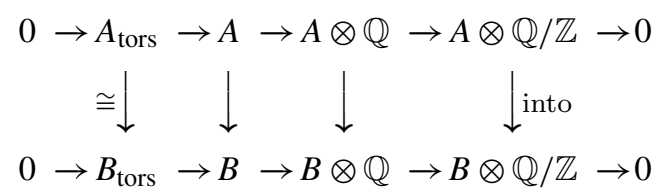


the map $A_{\text {tors }} \rightarrow B_{\text {tors }}$ is an isomorphism, and the map $A \otimes \mathbb{Q} / \mathbb{Z} \rightarrow B \otimes \mathbb{Q} / \mathbb{Z}$ is injective by (29). Hence $\operatorname{coker}\left(c_{\mathrm{L}, \mathrm{D}}^{m, n}\right)$ is torsion free; this proves (a). For $(\mathrm{b})$, we use that $\mathrm{CH}_{\mathrm{L}}^{n}(X)_{\text {tors }} \rightarrow$ $\mathrm{H}_{\mathrm{B}}^{2 n}(X, \mathbb{Z}(n))_{\text {tors }}$ is surjective by $(11)$. Hence the map $\mathrm{CH}_{\mathrm{L}}^{n}(X)_{\text {tors }} \rightarrow \mathrm{I}_{\mathrm{L}}^{2 n}(X)_{\text {tors }}$ is surjective, and the map $\mathrm{CH}_{\mathrm{L}}^{n}(X)_{\text {hom }} \otimes \mathbb{Q} / \mathbb{Z} \rightarrow \mathrm{CH}_{\mathrm{L}}^{n}(X) \otimes \mathbb{Q} / \mathbb{Z}$ is injective. It follows from this and (29) that the composition

$$
\mathrm{CH}_{\mathrm{L}}^{n}(X)_{\text {hom }} \otimes \mathbb{Q} / \mathbb{Z} \rightarrow \mathrm{CH}_{\mathrm{L}}^{n}(X) \otimes \mathbb{Q} / \mathbb{Z} \rightarrow \mathrm{H}_{\mathrm{D}}^{2 n}(X, \mathbb{Z}(n)) \otimes \mathbb{Q} / \mathbb{Z}
$$

is injective. But this composition coincides with

$$
\mathrm{CH}_{\mathrm{L}}^{n}(X)_{\text {hom }} \otimes \mathbb{Q} / \mathbb{Z} \rightarrow \mathrm{J}^{n}(X) \otimes \mathbb{Q} / \mathbb{Z} \rightarrow \mathrm{H}_{\mathrm{D}}^{2 n}(X, \mathbb{Z}(n)) \otimes \mathbb{Q} / \mathbb{Z},
$$

which is zero, since $\mathrm{J}^{n}(X) \otimes \mathbb{Q} / \mathbb{Z}=0$. This shows $\mathrm{CH}_{\mathrm{L}}^{n}(X)_{\text {hom }} \otimes \mathbb{Q} / \mathbb{Z}=0$. The last statement (c) is an easy consequence of (a). More precisely, if $\kappa^{n}: \mathrm{CH}^{n}(X) \rightarrow \mathrm{CH}_{\mathrm{L}}^{n}(X)$ is the comparison map, the restriction of the cycle map from Chow groups to Deligne-Beilinson cohomology to $\mathrm{CH}^{n}(X)_{\text {hom }}$ coincides with the Abel-Jacobi map $\Phi_{X}^{n}$, and $\mathrm{J}^{n}(X)$ is a direct factor of $\mathrm{H}_{\mathrm{D}}^{2 n}(X, \mathbb{Z}(n))$. Thus $0 \rightarrow \operatorname{ker}\left(\kappa^{n}\right) \rightarrow \operatorname{ker}\left(\Phi_{X}^{n}\right) \rightarrow \operatorname{ker}\left(c_{\mathrm{L}, \mathrm{D}}^{n}\right)$ is exact, where $\operatorname{ker}\left(\kappa^{n}\right)$ is a torsion group and $\operatorname{ker}\left(c_{\mathrm{L}, \mathrm{D}}^{n}\right)$ is torsion free by $(\mathrm{a})$.

Both Theorems 1.1 and 1.2 follow now from a formal argument using the existence of the cycle map $c_{\mathrm{L}, \mathrm{D}}^{m, n}$, together with the computations from Proposition 5.1 ; we use notations (25) and (26) from above.

Proof of Theorem 1.1. Consider the commutative diagram with exact rows

$$
\begin{aligned}
& 0 \rightarrow \mathrm{CH}_{\mathrm{L}}^{n}(X)_{\text {hom }} \rightarrow \mathrm{CH}_{\mathrm{L}}^{n}(X) \quad \rightarrow \quad \mathrm{I}_{\mathrm{L}}^{2 n}(X) \quad \rightarrow 0 \\
& c_{\mathrm{L}, \mathrm{D}}^{n} \mid \text { hom } \downarrow \quad c_{\mathrm{L}, \mathrm{D}}^{n} \downarrow \quad \downarrow \text { into } \\
& 0 \rightarrow \mathrm{J}^{n}(X) \quad \rightarrow \mathrm{H}_{\mathrm{D}}^{2 n}(X, \mathbb{Z}(n)) \rightarrow \operatorname{Hdg}^{2 n}(X, \mathbb{Z}) \rightarrow 0
\end{aligned}
$$

and the resulting short exact sequence

$$
0 \rightarrow \operatorname{coker}\left(c_{\mathrm{L}, \mathrm{D}}^{n} \mid \mathrm{hom}\right) \rightarrow \operatorname{coker}\left(c_{\mathrm{L}, \mathrm{D}}^{n}\right) \rightarrow \mathrm{Z}_{\mathrm{L}}^{2 n}(X) \rightarrow 0 .
$$

$\operatorname{Because} \operatorname{coker}\left(\left.c_{\mathrm{L}, \mathrm{D}}^{n}\right|_{\text {hom }}\right)$ is divisible as a quotient of $\mathbf{J}^{n}(X)$ and $\operatorname{coker}\left(c_{\mathrm{L}, \mathrm{D}}^{n}\right)$ is torsion free by Proposition 5.1(a), $\mathrm{Z}_{\mathrm{L}}^{2 n}(X)$ is torsion free. Using (27), we have

$$
\operatorname{HC}^{n}(X)_{\mathbb{Q}} \Leftrightarrow \mathrm{Z}^{2 n}(X) \otimes \mathbb{Q}=0 \Leftrightarrow \mathrm{Z}_{\mathrm{L}}^{2 n}(X)=0 \Leftrightarrow \mathrm{HC}_{\mathrm{L}}^{n}(X)_{\mathbb{Z}},
$$

which proves the first claim (a). The second claim (b) follows from (11).

Proof of Theorem 1.2. The first claim is proved in Proposition 5.1(a). For the second claim, we use that by Theorem 1.1 the integral L-Hodge conjecture is equivalent to the usual rational Hodge conjecture. Thus it suffices to show that

$$
\left.c_{\mathrm{L}, \mathrm{D}}^{2 n+1, n}\right|_{\text {tors }} \text { is injective } \Leftrightarrow \operatorname{HC}_{\mathrm{L}}^{n}(X)_{\mathbb{Z}} \text {. }
$$

Consider the commutative diagram with exact rows

$$
\begin{array}{ccc}
0 \rightarrow \quad \mathrm{CH}_{\mathrm{L}}^{n}(X) \otimes \mathbb{Q} / \mathbb{Z} & \rightarrow \mathrm{H}_{\mathrm{L}}^{2 n}(X, \mathbb{Q} / \mathbb{Z}(n)) \rightarrow \mathrm{H}_{\mathrm{L}}^{2 n+1}(X, \mathbb{Z}(n))_{\text {tors }} \rightarrow 0 \\
& \left.\cong \downarrow \quad \downarrow c_{\mathrm{L}, \mathrm{D}}^{2 n+1, n}\right|_{\text {tors }} \\
0 \rightarrow \mathrm{H}_{\mathrm{D}}^{2 n}(X, \mathbb{Z}(n)) \otimes \mathbb{Q} / \mathbb{Z} \rightarrow \mathrm{H}_{\mathrm{B}}^{2 n}(X, \mathbb{Q} / \mathbb{Z}(n)) & \rightarrow \mathrm{H}_{\mathrm{D}}^{2 n+1}(X, \mathbb{Z}(n))_{\text {tors }} \rightarrow 0
\end{array}
$$


Here, we have $\mathrm{CH}_{\mathrm{L}}^{n}(X) \otimes \mathbb{Q} / \mathbb{Z}=\mathrm{I}_{\mathrm{L}}^{2 n}(X) \otimes \mathbb{Q} / \mathbb{Z}$ by Proposition $5.1(\mathrm{~b})$, and $\mathrm{H}_{\mathrm{D}}^{2 n}(X, \mathbb{Z}(n)) \otimes$ $\mathbb{Q} / \mathbb{Z}=\operatorname{Hdg}^{2 n}(X, \mathbb{Z}) \otimes \mathbb{Q} / \mathbb{Z}$, since $\mathbf{J}^{n}(X)$ is divisible. Therefore the cokernel of the left vertical map is isomorphic to $\mathrm{Z}_{\mathrm{L}}^{2 n}(X) \otimes \mathbb{Q} / \mathbb{Z}$, and

$$
\operatorname{ker}\left(\left.c_{\mathrm{L}, \mathrm{D}}^{2 n+1, n}\right|_{\text {tors }}\right) \cong \mathrm{Z}_{\mathrm{L}}^{2 n}(X) \otimes \mathbb{Q} / \mathbb{Z} \text {. }
$$

Since $\mathrm{Z}_{\mathrm{L}}^{2 n}(X)$ is a finitely generated free abelian group, the group on the right is trivial if and only if $\mathrm{Z}_{\mathrm{L}}^{2 n}(X)=0$, i.e., if and only if the integral L-Hodge conjecture $\operatorname{HC}_{\mathrm{L}}^{n}(X)_{\mathbb{Z}}$ holds.

Remarks 5.2. (a) We state an integral $L$-version of the generalized Hodge conjecture [19]: recall that the level $l$ of a Hodge structure $0 \neq \mathrm{H}_{\mathbb{C}}=\oplus_{p+q=n} \mathrm{H}^{p, q}$ of weight $n$ is defined as $l=\max \left\{|p-q| \mid \mathrm{H}^{p, q} \neq 0\right\}$. For example, if $l=0$, then $n=2 p$ must be even, and $H$ is pure of type $(p, p)$. If $X$ is a smooth projective complex variety, we write $\operatorname{GHC}^{n}(X, c) \mathbb{Q}$ for the generalized Hodge conjecture in weight $n$ and level $n-2 c$. This is the statement that, for every $\mathbb{Q}$-Hodge substructure $\mathrm{H} \subseteq \mathrm{H}^{n}(X, \mathbb{Q})$ of level $n-2 c$, there exists a subvariety $Z \subseteq X$ of pure codimension $c$ such that $H$ is supported on $Z$, i.e., $\mathrm{H}=\operatorname{im}\left\{\mathrm{H}^{l}\left(Z_{0}, \mathbb{Q}(-c)\right) \rightarrow \mathrm{H}^{n}(X, \mathbb{Q})\right\}$, where $Z_{0} \rightarrow Z$ is a resolution of singularities. In weight $2 n$ and level $n$, the generalized Hodge conjecture says that the group of Hodge cycles $\operatorname{Hdg}^{2 n}(X, \mathbb{Q})$ is algebraic; thus $\operatorname{GHC}^{2 n}(X, n)_{\mathbb{Q}}$ is equivalent to the usual rational Hodge conjecture $\operatorname{HC}^{n}(X)_{\mathbb{Q}}$. In weight $2 n+1$ and level $n$, it is known that $\operatorname{GHC}^{2 n+1}(X, n)_{\mathbb{Q}}$ is equivalent to $\mathrm{HC}^{n}(C \times X)_{\mathbb{Q}}$ for all products of $X$ with a smooth projective curve $C$ [19, $\S 2]$.

Since for Lichtenbaum Chow groups we cannot argue with cycles directly, we use the following formulation of $\operatorname{GHC}^{n}(X, c) \mathbb{Q}$ in terms of correspondences; see, for example, [37, Lemma 0.1]: if $\mathrm{H} \subseteq \mathrm{H}^{n}(X, \mathbb{Q})$ is a $\mathbb{Q}$-Hodge substructure of level $l=n-2 c$, then $\operatorname{GHC}^{n}(X, c)_{\mathbb{Q}}$ holds for $\mathrm{H}$ if and only if there exists an integer $r$, and a smooth projective complex variety $Y$ of dimension $r-[(n-l) / 2]$, together with an element $z \in \mathrm{CH}^{r}(Y \times X)$ such that $\mathrm{H}$ is contained in $z_{*} \mathrm{H}^{l}(Y, \mathbb{Q})$. Here, $z_{*}$ is given by the usual formula for the action of correspondences:

$$
\left.z_{*}: \mathrm{H}^{l}(Y, \mathbb{Q})\right) \rightarrow \mathrm{H}^{n}(X, \mathbb{Q}), \quad \eta \mapsto \operatorname{pr}_{X *}\left(\operatorname{pr}_{Y}^{*}(\eta) \cup c^{p}(z)\right) .
$$

We write $\operatorname{GHC}_{\mathrm{L}}^{n}(X, c)_{\mathbb{Z}}$ for the statement that, for every $\mathbb{Z}$-Hodge substructure $\mathrm{H} \subseteq$ $\mathrm{H}^{n}(Z, \mathbb{Z})$ of level $l=n-2 c$, there exists an integer $r$, a smooth projective complex variety $Y$, and an element $z \in \mathrm{CH}_{\mathrm{L}}^{r}(Y \times X)$, such that $\mathrm{H}$ is contained in $z_{*} \mathrm{H}$, where $z_{*} \mathrm{H}^{l}(Y, \mathbb{Z}) \rightarrow$ $\mathrm{H}^{n}(X, \mathbb{Z})$ is defined by the above formula, replacing the usual cycle map $c^{p}$ by its L-version $c_{\mathrm{L}}^{p}$. We can show that

$$
\mathrm{GHC}^{2 n-1}(X, n-1)_{\mathbb{Q}}=\operatorname{GHC}_{\mathrm{L}}^{2 n-1}(X, n-1)_{\mathbb{Z}} \quad \text { for } n \geqslant 1,
$$

which can be deduced from Theorem 1.1.

(b) Let $X$ be a smooth projective complex variety. Our results have the following consequence for the unramified cohomology group $\mathrm{H}_{\mathrm{nr}}^{4}(X, \mathbb{Q} / \mathbb{Z}(3))$. Let $\alpha: X_{\text {ét }} \rightarrow$ $X_{\text {Zar }}$, and assume that $\operatorname{dim} X \leqslant 5$, so that in the Leray spectral sequence $\mathrm{E}_{2}^{r, s}=$ $\mathrm{H}^{r}\left(X, R^{s} \alpha_{*}\left(\mathbb{Z}_{X}(3)\right)_{\text {ét }}\right)=0$ for $r \geqslant 6$. The spectral sequence yields the exact sequence (see 
also [26, Remarques 2.10])

$$
0 \rightarrow \mathrm{H}_{\mathrm{M}}^{5}(X, \mathbb{Z}(3)) \rightarrow \mathrm{H}_{\mathrm{L}}^{5}(X, \mathbb{Z}(3)) \stackrel{\Psi}{\rightarrow} \mathrm{H}_{\mathrm{nr}}^{4}(X, \mathbb{Q} / \mathbb{Z}(3)) \stackrel{\Theta}{\rightarrow} \mathrm{CH}^{3}(X) \stackrel{\kappa^{3}}{\rightarrow} \mathrm{CH}_{\mathrm{L}}^{3}(X) .
$$

Here $\Psi$ is the edge map $\mathrm{H}_{\mathrm{L}}^{5}(X, \mathbb{Z}(3)) \rightarrow \mathrm{H}^{0}\left(X, R^{5} \alpha_{*} \mathbb{Z}_{X}(2)_{\text {ét }}\right)$, and the map $\Theta$ is the $d_{3}$-differential $d_{3}^{0,5}$, which can be shown to coincide with the composition

$$
\begin{aligned}
\mathrm{H}_{\mathrm{nr}}^{4}(X, \mathbb{Q} / \mathbb{Z}(3)) & \stackrel{d_{2}^{0,4}}{\rightarrow} \mathrm{H}^{2}\left(X, \mathcal{H}_{B}^{3}(\mathbb{Q} / \mathbb{Z}(3)) \stackrel{\cong}{\cong} \mathrm{H}^{2}\left(X, \mathcal{H}_{M}^{3}(\mathbb{Q} / \mathbb{Z}(3))\right)\right. \\
& \stackrel{\cong}{\rightarrow} \mathrm{H}_{\mathrm{M}}^{5}(X, \mathbb{Q} / \mathbb{Z}(3)) \rightarrow \mathrm{CH}^{3}(X)_{\text {tors }},
\end{aligned}
$$

where the first isomorphism is induced from the isomorphism of Zariski sheaves $\mathcal{H}_{M}^{3}(\mathbb{Q} / \mathbb{Z}(3)) \stackrel{\cong}{\rightarrow} \mathcal{H}_{B}^{3}(\mathbb{Q} / \mathbb{Z}(3))$, the second isomorphism comes from the coniveau spectral sequence for motivic cohomology (this uses that $\mathcal{H}_{M}^{q}(\mathbb{Q} / \mathbb{Z}(3))=0$ for $q>3$ ), and the last map is the map in the universal coefficient sequence for motivic cohomology. By Proposition 5.1(c) $\operatorname{ker}\left(\kappa^{3}\right)=\operatorname{ker}\left(\Phi_{X}^{3}\right)_{\text {tors }}$, there is thus a surjective map:

$$
\Psi: \mathrm{H}_{\mathrm{nr}}^{4}(X, \mathbb{Q} / \mathbb{Z}(3)) \rightarrow \operatorname{ker}\left(\Phi_{X}^{3}\right)_{\text {tors }}
$$

The examples from 3.2(c) of 4 -folds with $\# \mathrm{CH}^{3}(X)_{\text {tors }}=\infty$ are constructed using product cycles which have trivial image under the Abel-Jacobi map $\Phi_{X}^{3}$. In particular, in these examples $\operatorname{ker}\left(\Phi_{X}^{3}\right)_{\text {tors }}$ contains a subgroup of infinite corank, and thus $\mathrm{H}_{\mathrm{nr}}^{4}(X, \mathbb{Q} / \mathbb{Z}(3))$ can be very 'large'. We note that, if $\bar{\Phi}_{X}^{3}$ is the map induced by the Abel-Jacobi map on the quotient $\mathrm{H}^{3}\left(X, \mathcal{H}_{B}^{3}(\mathbb{Z}(3))\right)$ of $\mathrm{CH}^{3}(X)$ (i.e., the group of codimension 3 cycles modulo cycles algebraically equivalent to zero), the composition of $\Psi$ with the surjective map $\operatorname{ker}\left(\Phi_{X}^{3}\right)_{\text {tors }} \rightarrow \operatorname{ker}\left(\Phi_{X}^{3}\right)_{\text {tors }}$ is the map considered by Voisin in [55].

\section{Tate conjecture}

Let $k$ be a field, finitely generated over the prime field, and let $X$ be a smooth projective geometrically irreducible $k$-variety. If $\bar{k}$ is a separable closure of $k$, let $\bar{X}=X \times_{k} \bar{k}$, and let $\Gamma_{k}=\operatorname{Gal}(\bar{k} / k)$. If $\ell \neq \operatorname{char}(k)$, we have a cycle map from Lichtenbaum to continuous étale cohomology (see $[25, \S 1.4]$ and $[26, \S 3.1]$ )

$$
\mathrm{H}_{\mathrm{L}}^{m}(X, \mathbb{Z}(n)) \otimes \mathbb{Z}_{\ell} \rightarrow \mathrm{H}_{\text {cont }}^{m}\left(X, \mathbb{Z}_{\ell}(n)\right)
$$

whose composite with the map $\pi^{m, n}: \mathrm{H}_{\text {cont }}^{m}\left(X, \mathbb{Z}_{\ell}(n)\right) \rightarrow \mathrm{H}_{\text {ét }}^{m}\left(\bar{X}, \mathbb{Z}_{\ell}(n)\right)^{\Gamma_{k}}$ from the Hochschild-Serre spectral sequence $[22,(0.3)]$ defines a cycle map

$$
c_{\mathrm{L}, \mathbb{Z}_{\ell}}^{m, n}: \mathrm{H}_{\mathrm{L}}^{m}(X, \mathbb{Z}(n)) \otimes \mathbb{Z}_{\ell} \rightarrow \mathrm{H}_{\mathrm{ét}}^{m}\left(\bar{X}, \mathbb{Z}_{\ell}(n)\right)^{\Gamma_{k}},
$$

which tensored with $\mathbb{Q}_{\ell}$ coincides with the map considered by Tate. We remark that (30) arises from a morphism in the derived category $\mathrm{D}^{-}\left(\mathrm{AbShv}_{\text {ét }}\left(X_{\text {ét }}\right)\right)$

$$
\left.\mathbb{Z}_{X}(n)_{\text {ét }}^{\stackrel{L}{\otimes}} \mathbb{Z}_{\ell} \rightarrow \mathbb{Z}_{\ell}^{c}(n)\right|_{X}
$$

Assume now that $k \subseteq \mathbb{C}$. Recall that we write $\mathrm{TC}^{n}(\bar{X})_{\mathbb{Q}_{\ell}}$ for the assertion that, if $k$ is finitely generated over $\mathbb{Q}$, the cycle map (4) is surjective. We can state an integral L-version $\operatorname{TC}_{\mathrm{L}}^{n}(\bar{X})_{\mathbb{Z}_{\ell}}$ of this as follows. We have an isomorphism

$$
\mathrm{CH}_{\mathrm{L}}^{n}(\bar{X}) \cong \lim _{\rightarrow} \mathrm{CH}_{\mathrm{L}}^{n}\left(X_{k^{\prime}}\right)
$$


where the limit is taken over all extensions $k \subseteq k^{\prime} \subseteq \bar{k}$ of finite degree over $k$. Thus taking the direct limit over the $c_{\mathrm{L}, \mathbb{Z}_{\ell}}^{n}=c_{\mathrm{L}, \mathbb{Z}_{\ell}}^{2 n, n}$ from (31), we obtain a map

$$
\bar{c}_{\mathrm{L}, \mathbb{Z}_{\ell}}^{n}: \mathrm{CH}_{\mathrm{L}}^{n}(\bar{X}) \otimes \mathbb{Z}_{\ell} \rightarrow \operatorname{Ta}^{2 n}\left(\bar{X}, \mathbb{Z}_{\ell}\right)=\lim _{\rightarrow} \mathrm{H}_{\text {ét }}^{2 n}\left(\bar{X}, \mathbb{Z}_{\ell}(n)\right)^{\Gamma_{k^{\prime}}},
$$

and the integral L-Tate conjecture $\mathrm{TC}_{\mathrm{L}}^{n}(\bar{X})_{\mathbb{Z}_{\ell}}$ is the claim that this map $\bar{c}_{\mathrm{L}, \mathbb{Z}_{\ell}}$ is surjective. We now prove Theorem 1.3:

Proof of Theorem 1.3. Let $\mathrm{I}_{\mathrm{L}}^{2 n}(\bar{X})_{\mathbb{Z}_{\ell}}$ be the image of the map (33), and let

$$
\mathrm{Z}_{\mathrm{L}}^{2 n}(\bar{X})_{\mathbb{Z}_{\ell}}=\operatorname{Ta}^{2 n}\left(\bar{X}, \mathbb{Z}_{\ell}\right) / \mathrm{I}_{\mathrm{L}}^{2 n}(\bar{X})_{\mathbb{Z}_{\ell}}
$$

be the obstruction to the integral L-Tate conjecture. We need to show that $\mathrm{Z}_{\mathrm{L}}^{2 n}(\bar{X})_{\mathbb{Z}_{\ell}}$ is torsion free. Consider first $X_{\mathbb{C}}=X \times_{k} \mathbb{C}$. As before, let $\mathrm{I}_{\mathrm{L}}^{2 n}\left(X_{\mathbb{C}}\right)$ be the image of the cycle map $\mathrm{CH}_{\mathrm{L}}^{n}\left(X_{\mathbb{C}}\right) \rightarrow \mathrm{H}_{\mathrm{B}}^{2 n}\left(X_{\mathbb{C}}, \mathbb{Z}(n)\right)$. The proof of Theorem 1.1 shows that the quotient $\mathrm{Z}_{\mathrm{L}}^{2 n}\left(X_{\mathbb{C}}\right)=\operatorname{Hdg}^{2 n}\left(X_{\mathbb{C}}, \mathbb{Z}\right) / \mathrm{I}_{\mathrm{L}}^{2 n}\left(X_{\mathbb{C}}\right)$ is torsion free. Since, by definition, we have $\operatorname{Hdg}^{2 n}\left(X_{\mathbb{C}}, \mathbb{Z}\right)_{\text {tors }}=\mathrm{H}_{\mathrm{B}}^{2 n}\left(X_{\mathbb{C}}, \mathbb{Z}(n)\right)_{\text {tors }}$, it follows that the quotient $\mathrm{H}_{\mathrm{B}}^{2 n}\left(X_{\mathbb{C}}, \mathbb{Z}(n)\right) / \operatorname{Hdg}^{2 n}\left(X_{\mathbb{C}}, \mathbb{Z}\right)$ is torsion free as well. Hence

$$
\left(\mathrm{H}_{\mathrm{B}}^{2 n}\left(X_{\mathbb{C}}, \mathbb{Z}(n)\right) / \mathrm{I}_{\mathrm{L}}^{2 n}\left(X_{\mathbb{C}}\right)\right)_{\text {tors }}=0 .
$$

Furthermore, the cycles maps to $\ell$-adic and singular cohomology are clearly compatible with the comparison isomorphism in cohomology, and thus the square

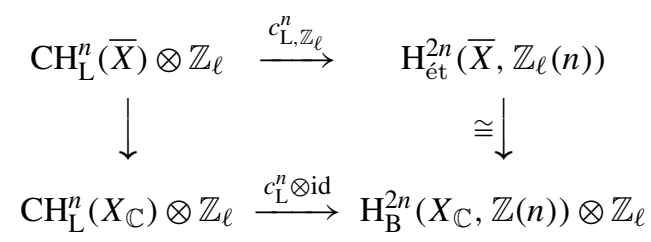

commutes, and we have an inclusion $\mathrm{I}_{\mathrm{L}}^{2 n}(\bar{X})_{\mathbb{Z}_{\ell}} \rightarrow \mathrm{I}_{\mathrm{L}}^{2 n}\left(X_{\mathbb{C}}\right) \otimes \mathbb{Z}_{\ell}$. We claim that this inclusion is an isomorphism; given this, we obtain for the cokernels

$$
\mathrm{H}_{\text {ét }}^{2 n}\left(\bar{X}, \mathbb{Z}_{\ell}(n)\right) / \mathrm{I}_{\mathrm{L}}^{2 n}(\bar{X})_{\mathbb{Z}_{\ell}} \cong\left(\mathrm{H}_{\mathrm{B}}^{2 n}\left(X_{\mathbb{C}}, \mathbb{Z}(n)\right) / \mathrm{I}_{\mathrm{L}}^{2 n}\left(X_{\mathbb{C}}\right)\right) \otimes \mathbb{Z}_{\ell},
$$

where, by (34), the group on the right has no torsion. Hence the group on the left is torsion free, and the same holds for the subgroup $\mathrm{Z}_{\mathrm{L}}^{2 n}(\bar{X})_{\mathbb{Z}_{\ell}}$, as claimed.

To show that $\mathrm{I}_{\mathrm{L}}^{2 n}(\bar{X})_{\mathbb{Z}_{\ell}} \rightarrow \mathrm{I}_{\mathrm{L}}^{2 n}\left(X_{\mathbb{C}}\right) \otimes \mathbb{Z}_{\ell}$ is surjective, it suffices to show that, if $\bar{k} \subseteq$ $K \subseteq \mathbb{C}$ is an extension of algebraically closed fields, then under the canonical isomorphism

$$
\mathrm{H}_{\mathrm{ét}}^{2 n}\left(\bar{X}, \mathbb{Z}_{\ell}(n)\right) \stackrel{\cong}{\rightarrow} \mathrm{H}_{\mathrm{ét}}^{2 n}\left(X_{K}, \mathbb{Z}_{\ell}(n)\right)
$$

the images of $\mathrm{CH}_{\mathrm{L}}^{n}(\bar{X}) \otimes \mathbb{Z}_{\ell}$ and $\mathrm{CH}_{\mathrm{L}}^{n}\left(X_{K}\right) \otimes \mathbb{Z}_{\ell}$ coincide. For this, we write $K$ as a direct limit of finitely generated smooth $\bar{k}$-algebras $A$, which yields

$$
\mathrm{CH}_{\mathrm{L}}^{n}\left(X_{K}\right) \cong \lim _{\rightarrow} \mathrm{CH}_{\mathrm{L}}^{n}\left(\bar{X} \times_{\bar{k}} A\right)
$$

Hence it suffices to note that $\mathrm{CH}_{\mathrm{L}}^{n}(\bar{X}) \otimes \mathbb{Z}_{\ell}$ and $\mathrm{CH}_{\mathrm{L}}^{n}\left(\bar{X} \times_{\bar{k}} A\right) \otimes \mathbb{Z}_{\ell}$ have the same image in $\mathrm{H}_{\text {ét }}^{2 n}\left(X_{K}, \mathbb{Z}_{\ell}(n)\right)$, which follows from an easy specialization argument to a closed point of $\operatorname{Spec}(A)$. The remaining claim that $\operatorname{Ta}^{2 n}\left(\bar{X}, \mathbb{Z}_{\ell}\right)$ tors is contained in the 
image of $\mathrm{CH}_{\mathrm{L}}^{n}(\bar{X})_{\text {tors }} \otimes \mathbb{Z}_{\ell}$ follows easily from the above, together with the proof of Proposition 3.1.

If $k$ is a finite field, we consider the integral L-Tate conjecture in the form

$$
\operatorname{TC}_{\mathrm{L}}^{n}(X)_{\mathbb{Z}_{\ell}} \Leftrightarrow \operatorname{im}\left(c_{\mathrm{L}, \mathbb{Z}_{\ell}}^{n}\right)=\mathrm{H}_{\text {ét }}^{2 n}\left(\bar{X}, \mathbb{Z}_{\ell}(n)\right)^{\Gamma_{k}} .
$$

We now prove Theorem 1.4. We remark that the second part of the proof is similar to an argument by Jannsen [23, p. 328], who used the comparison map between Quillen and étale $K$-theory with finite coefficients to motivate the surjectivity of his $\ell$-adic regulator map.

Proof of Theorem 1.4. We show first that $\mathrm{TC}^{n}(X)_{\mathbb{Q}_{\ell}} \Leftrightarrow \operatorname{TC}_{\mathrm{L}}^{n}(X)_{\mathbb{Z}_{\ell}}$. Consider

$$
\mathrm{Z}_{\mathrm{L}, \mathbb{Z}_{\ell}}^{2 n}(X)=\operatorname{coker}\left\{c_{\mathrm{L}, \mathbb{Z}_{\ell}}^{n}: \mathrm{CH}_{\mathrm{L}}^{n}(X) \otimes \mathbb{Z}_{\ell} \rightarrow \mathrm{H}_{\text {ét }}^{2 n}\left(\bar{X}, \mathbb{Z}_{\ell}(n)\right)^{\Gamma_{k}}\right\}
$$

i.e., the obstruction to the integral Tate conjecture $\operatorname{TC}_{\mathrm{L}}^{n}(X)_{\mathbb{Z}_{\ell}}$; thus the usual Tate conjecture $\mathrm{TC}^{n}(X)_{\mathbb{Q}_{\ell}}$ holds if and only if $\mathrm{Z}_{\mathrm{L}, \mathbb{Z}_{\ell}}^{2 n}(X)$ is a finite group. Let $\mathrm{K}_{X}(p)$ ét be a cone of the morphism (32). Since tensored with $\mathbb{Z} / \ell^{r} \mathbb{Z}$ the morphism (32) is an isomorphism, the hypercohomology groups of $\mathrm{K}_{X}(p)$ ét are uniquely $\ell$-divisible [26, Proposition 3.4]. It follows that the cokernel

$$
\mathrm{Q}_{\mathrm{L}, \mathbb{Z}_{\ell}}^{2 n}(X)=\operatorname{coker}\left\{\mathrm{CH}_{\mathrm{L}}^{n}(X) \otimes \mathbb{Z}_{\ell} \rightarrow \mathrm{H}_{\text {cont }}^{2 n}\left(X, \mathbb{Z}_{\ell}(n)\right)\right\}
$$

satisfies $\mathrm{Q}_{\mathrm{L}, \mathbb{Z}_{\ell}}^{2 n}(X)\{\ell\}=0$. In the commutative diagram with exact rows

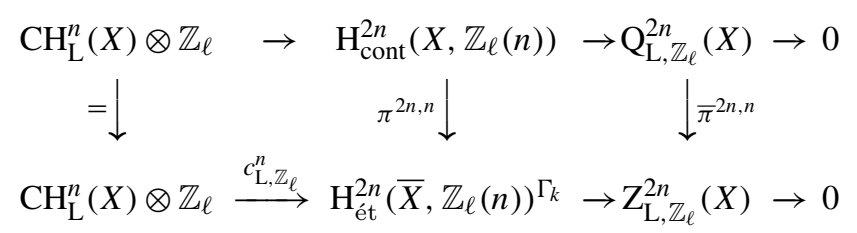

the map $\pi^{2 n, n}$ is surjective, since $\operatorname{cd}_{\ell}(k)=1$ for a finite field. Hence the finitely generated $\mathbb{Z}_{\ell}$-module $\mathrm{Z}_{\mathrm{L}, \mathbb{Z}_{\ell}}^{2 n}(X)$ is a quotient of $\mathrm{Q}_{\mathrm{L}, \mathbb{Z}_{\ell}}^{2 n}(X)$. Furthermore,

$$
\operatorname{ker}\left(\pi^{2 n, n}\right)=\mathrm{H}^{1}\left(\Gamma_{k}, \mathrm{H}_{\text {ét }}^{2 n-1}\left(\bar{X}, \mathbb{Z}_{\ell}(n)\right)\right),
$$

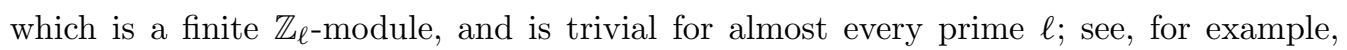
$\left[12\right.$, Proof of Théorème 2]. Since $\mathrm{Q}_{\mathrm{L}, \mathbb{Z}_{\ell}}^{2 n}(X)\{\ell\}=0$, the induced map $\operatorname{ker}\left(\pi^{2 n, n}\right) \rightarrow \mathrm{Q}_{\mathrm{L}, \mathbb{Z}_{\ell}}^{2 n}$ is trivial, and $\mathrm{Q}_{\mathrm{L}, \mathbb{Z}_{\ell}}^{2 n}(X) \cong \mathrm{Z}_{\mathrm{L}, \mathbb{Z}_{\ell}}^{2 n}(X)$; in particular, $\mathrm{Z}_{\mathrm{L}, \mathbb{Z}_{\ell}}^{2 n}(X)$ is a finitely generated free $\mathbb{Z}_{\ell}$-module. From this, we immediately obtain that

$$
\operatorname{TC}^{n}(X)_{\mathbb{Q}_{\ell}} \Leftrightarrow \mathrm{Z}_{\mathrm{L}, \mathbb{Z}_{\ell}}^{2 n}(X) \otimes \mathbb{Q}=0 \Leftrightarrow \mathrm{Z}_{\mathrm{L}, \mathbb{Z}_{\ell}}^{2 n}(X)=0 \Leftrightarrow \mathrm{TC}_{\mathrm{L}}^{n}(X)_{\mathbb{Z}_{\ell}} .
$$

For the remaining equivalence, we use the basic fact that, for an abelian group $A$, a finitely generated $\mathbb{Z}_{\ell}$-module $B$, and a homomorphism $f: A \rightarrow B$, the induced map $A \otimes_{\mathbb{Z}} \mathbb{Z}_{\ell} \rightarrow B$ is surjective if and only if the induced map on the $\ell$-completion $A^{\wedge} \rightarrow B$ is surjective. We applied this to $A=\mathrm{CH}_{\mathrm{L}}^{n}(X), B=\mathrm{H}_{\text {ét }}^{2 n}\left(\bar{X}, \mathbb{Z}_{\ell}(n)\right)^{\Gamma_{k}}$, and the map $c$ : 
$\mathrm{CH}_{\mathrm{L}}^{n}(X) \rightarrow \mathrm{H}_{\text {ét }}^{2 n}\left(\bar{X}, \mathbb{Z}_{\ell}(n)\right)^{\Gamma_{k}}$ given by the composite of $\mathrm{CH}_{\mathrm{L}}^{n}(X) \rightarrow \mathrm{CH}_{\mathrm{L}}^{n}(\bar{X})$ with the $\ell$-adic cycle map (9) for $\bar{X}$. Since $c$ induces on $\mathrm{CH}_{\mathrm{L}}^{n}(X) \otimes \mathbb{Z}_{\ell}$ the cycle map $\bar{c}_{\mathrm{L}, \mathbb{Z}_{\ell}}^{n}$, we obtain

$$
\mathrm{TC}_{\mathrm{L}}^{n}(X)_{\mathbb{Z}_{\ell}} \Leftrightarrow c^{\wedge}: \mathrm{CH}_{\mathrm{L}}^{n}(X)^{\wedge} \rightarrow \mathrm{H}_{\mathrm{et}}^{2 n}\left(\bar{X}, \mathbb{Z}_{\ell}(n)\right)^{\Gamma_{k}} \text { is surjective. }
$$

We use again that over a finite field the map $\pi^{2 n, n}$ is surjective. The diagram

$$
\begin{aligned}
0 \rightarrow \lim _{\leftarrow}^{1} \mathrm{H}_{\text {ét }}^{2 n-1}\left(X, \mu_{\ell^{v}}^{\otimes n}\right) \rightarrow & \mathrm{H}_{\text {cont }}^{2 n}\left(X, \mathbb{Z}_{\ell}(n)\right) \rightarrow \lim _{\leftarrow} \mathrm{H}_{\text {ét }}^{2 n}\left(X, \mu_{\ell^{r}}^{\otimes n}\right) \rightarrow 0 \\
& \pi^{2 n, n} \downarrow \\
& \mathrm{H}_{\text {ét }}^{2 n}\left(\bar{X}, \mathbb{Z}_{\ell}(n)\right)^{\Gamma_{k}} \stackrel{\cong}{\cong} \lim _{\leftarrow} \mathrm{H}_{\text {ét }}^{2 n, n}\left(\bar{X}, \mu_{\ell^{r}}^{\otimes n}\right)^{\Gamma_{k}}
\end{aligned}
$$

commutes, and the fact that $\pi^{2 n, n}$ is surjective implies that $\bar{\pi}^{2 n, n}$ is surjective.

On the other hand, from the exact sequence of finite abelian groups

$$
0 \rightarrow \mathrm{CH}_{\mathrm{L}}^{n}(X) \otimes \mathbb{Z} / \ell^{r} \rightarrow \mathrm{H}_{\text {ét }}^{2 n}\left(X, \mu_{\ell^{r}}^{\otimes n}\right) \rightarrow \mathrm{H}_{\mathrm{L}}^{2 n+1}(X, \mathbb{Z}(n))\left[\ell^{r}\right] \rightarrow 0,
$$

we obtain, taking the inverse limit, the exact sequence

$$
0 \rightarrow \mathrm{CH}_{\mathrm{L}}^{n}(X)^{\wedge} \rightarrow \lim _{\leftarrow} \mathrm{H}_{\text {ét }}^{2 n}\left(X, \mu_{\ell^{r}}^{\otimes n}\right) \rightarrow \mathrm{T}_{\ell} \mathrm{H}_{\mathrm{L}}^{2 n+1}(X, \mathbb{Z}(n)) \rightarrow 0,
$$

which fits into the following commutative diagram with exact rows:

$$
\begin{array}{cccc}
\mathrm{CH}_{\mathrm{L}}^{n}(X)^{\wedge} & \rightarrow \underset{\leftarrow}{\lim } \mathrm{H}_{\text {ét }}^{2 n}\left(X, \mu_{\ell^{r}}^{\otimes n}\right) & \rightarrow \mathrm{T}_{\ell} \mathrm{H}_{\mathrm{L}}^{2 n+1}(X, \mathbb{Z}(n)) \rightarrow 0 \\
=\downarrow & \bar{\pi}^{2 m, m} \downarrow \text { onto } & \downarrow
\end{array}
$$

Here, $\operatorname{ker}\left(\bar{\pi}^{2 n, n}\right)$ is a quotient of the finite group $\mathrm{H}^{1}\left(\Gamma_{k}, \mathrm{H}_{\text {ét }}^{2 n-1}\left(\bar{X}, \mathbb{Z}_{\ell}(n)\right)\right)$. Since the Tate module $\mathrm{T}_{\ell} \mathrm{H}_{\mathrm{L}}^{2 n+1}(X, \mathbb{Z}(n))$ is torsion free, the map $\bar{\pi}^{2 n, n}$ induces an isomorphism $\mathrm{T}_{\ell} \mathrm{H}_{\mathrm{L}}^{2 n+1}(X, \mathbb{Z}(n)) \cong \operatorname{coker}\left(c^{\wedge}\right)$, which shows that

$$
\mathrm{TC}_{\mathrm{L}}^{n}(X)_{\mathbb{Z}_{\ell}} \Leftrightarrow \mathrm{T}_{\ell} \mathrm{H}_{\mathrm{L}}^{2 n+1}(X, \mathbb{Z}(n))=0 .
$$

The $\ell^{r}$-torsion subgroup $\mathrm{H}_{\mathrm{L}}^{2 n+1}(X, \mathbb{Z}(n))\left[\ell^{r}\right]$ is a quotient of the finite group $\mathrm{H}_{\text {ét }}^{2 n}\left(X, \mu_{\ell^{r}}^{\otimes n}\right)$. Hence $\mathrm{H}_{\mathrm{L}}^{2 n+1}(X, \mathbb{Z}(n))\{\ell\}=\left(\mathbb{Q}_{\ell} / \mathbb{Z}_{\ell}\right)^{\rho(X, \ell)} \oplus F$, where $F$ is a finite group and $\rho(X, \ell)$ is a non-negative integer. It follows that the Tate module $\mathrm{T}_{\ell} \mathrm{H}_{\mathrm{L}}^{2 n+1}(X, \mathbb{Z}(n))$ is a free $\mathbb{Z}_{\ell}$-module of rank $\rho(X, \ell)$; in particular,

$$
\mathrm{TC}_{\mathrm{L}}^{n}(X)_{\mathbb{Z}_{\ell}} \Leftrightarrow \mathrm{H}_{\mathrm{L}}^{2 n+1}(X, \mathbb{Z}(n))\{\ell\}<\infty .
$$

The remaining claim that the torsion in $\mathrm{H}_{\text {ét }}^{2 n}\left(\bar{X}, \mathbb{Z}_{\ell}(n)\right)^{\Gamma_{k}}$ is L-algebraic follows from the fact that $\mathrm{T}_{\ell} \mathrm{H}_{\mathrm{L}}^{2 n+1}(X, \mathbb{Z}(n)) \cong \operatorname{coker}\left(c^{\wedge}\right)$ is torsion free. 
Acknowledgements. The authors thank J. Ayoub, S. Bloch, J. Colliot-Thélène, M. Levine, and S. Lichtenbaum for discussions. The first author gratefully acknowledges the hospitality of the Tata Institute of Fundamental Research. The second author was supported during this work by a J. C. Bose Fellowship from the Department of Science and Technology, India.

\section{References}

1. M. Artin And B. Mazur, Etale Homotopy, Lecture Notes in Mathematics, Volume 100 (Springer, Berlin, 1986).

2. M. F. Atiyah and F. Hirzebruch, Analytic cycles on complex manifolds, Topology 1 (1962), 25-45.

3. S. Bloch And A. Ogus, Gersten's conjecture and the homology of schemes, Ann. Sci. Éc. Norm. Supér (4) 7 (1974), 181-201.

4. S. Bloch, Algebraic $K$-theory and crystalline cohomology, Publ. Math. Inst. Hautes Études Sci. 47 (1977), 187-268.

5. S. BlOCH, Torsion algebraic cycles and a theorem of Roitman, Compositio Math. 39 (1979), 107-127.

6. S. BlOCH, Algebraic cycles and higher K-theory, Adv. Math. 61 (1986), 267-304.

7. S. Bloch, Algebraic cycles and the Berlinson conjectures, in The Lefschetz Centennial Conference, Part I (Mexico City, 1984), Contemparary Mathematics, Volume 58, pp. 65-79 (American Mathematical Society, Providence, RI, 1986).

8. S. BLOCH, 1994. Some notes on elementary properties of higher Chow groups (unpublished note).

9. S. Bloch, The moving lemma for higher Chow groups, J. Algebraic Geom. 3 (1994), $537-568$.

10. K. S. Brown And S. M. Gersten, Algebraic $K$-theory as generalized sheaf cohomology, in Algebraic K-Theory, I: Higher K-Theories (Proc. Conf., Battelle Memorial Inst., Seattle, WA, 1972), Lecture Notes in Mathematics, Volume 341, pp. 266-292 (Springer, Berlin, 1973).

11. J.-L. Colliot-Thélène And B. Kahn, Cycles de codimension 2 et $H^{3}$ non ramifié pour les variétés sur les corps finis, J. K-Theory 11 (2013), 1-53.

12. J.-L. Colliot-Thélène, J.-J. Sansuc and C. Soulé, Torsion dans le groupe de Chow de codimension deux, Duke Math. J. 50 (1983), 763-801.

13. J.-L. Colliot-Thélène And T. Szamuely, Autour de la conjecture de Tate à coefficients $\mathbf{Z}_{\ell}$ pour les variétés sur les corps finis, in The Geometry of Algebraic Cycles, Clay Mathematics Proceedings, Volume 9, pp. 83-98 (American Mathematical Society, 2010).

14. J.-L. Colliot-ThélÈne And C. Voisin, Cohomologie non ramifée conjecture de Hodge entière, Duke Math. J. 161 (2012), 735-801.

15. H. Esnault and E. Viehweg, Deligne-Beulinson cohomology, in Bellinson's Conjectures on special values of L-functions, Perspect Math., Volume 4, pp. 43-91 (Academic Press, Boston, MA, 1988).

16. T. Geisser, Tate's conjecture, algebraic cycles and rational $K$-theory, $K$-Theory $\mathbf{1 3}$ (1998), 109-128.

17. T. Geisser and M. Levine, The $K$-theory of fields in characteristic $p$, Invent. Math 139 (2000), 459-493.

18. T. Geisser And M. Levine, The Bloch-Kato conjecture and a theorem of Suslin-Voevodsky, J. Reine Angew. Math. 530 (2001), 55-103. 
19. A. Grothendieck, Hodge's general conjecture is false for trivial reasons, Topology 8 (1969), 299-303.

20. W. V. D. Hodge, The topological invariants of algebraic varieties, in Proceedings of the International Congress of Mathematicians, Cambridge, MA, 1950, Volume 1, pp. 182-192 (American Mathematical Society, Providence, RI, 1952).

21. L. Illusie, Complexes de de Rham-Witt et cohomologie cristalline, Ann. Sci. Éc. Norm. Supér 12 (1979), 501-661.

22. U. Jannsen, Continuous étale cohomology, Math. Ann. 280 (1988), 207-245.

23. U. Jannsen, On the $l$-adic cohomology of varieties over number fields and its Galois cohomology, in Galois Groups Over Q (Berkeley, CA, 1987), Mathematical Sciences Research Institute Publications, Volume 16, pp. 315-360 (Springer, New York, 1989).

24. B. KAHn, Applications of weight-two motivic cohomology, Doc. Math. 1 (1996), 395-416.

25. B. KAHN, The Geisser-Levine method revisited and algebraic cycles over a finite field, Math. Ann. 324 (2002), 581-617.

26. B. Kahn, Classes de cycles motiviques étales, Algebra Number Theory 6 (2012), 1369-1407.

27. J. Kollar, Trento examples, in Classification of Irregular Varieties (Trento, 1990), Lecture Notes in Mathematics, Volume 1515, pp. 134-139 (Springer, Berlin, 1992).

28. M. Levine, Chow's moving lemma and the homotopy coniveau tower, $K$-Theory 37 (2006), 129-209.

29. M. Levine, Mixed Motives, Math. Surveys and Monographs, Volume 57 (American Mathematical Society, Providence, RI, 1992).

30. S. Lichtenbaum, The construction of weight-two arithmetic cohomology, Invent. Math. 88 (1987), 183-215.

31. S. Lichtenbaum, New results on weight-two motivic cohomology, in The Grothendieck Festschrift, Vol. III, Progress in Mathematics, Volume 88, pp. 35-55 (Birkhäuser, Boston, MA, 1990).

32. A. S. Merkurjev AND A. A. Suslin, $K$-cohomology of Severi-Brauer varieties and the norm residue homomorphism, Izv. Akad. Nauk SSSR Ser. Mat. 46 (1982), 1011-1046, 1135-1136.

33. A. S. Merkurjev and A. A. Suslin, Norm residue homomorphism of degree three, Izv. Akad. Nauk SSSR Ser. Mat. 54 (1990), 339-356.

34. J. S. Milne, Motivic cohomology and values of zeta functions, Compositio Math. 68 (1988), 59-100.

35. M. V. NorI, Algebraic cycles and Hodge-theoretic connectivity, Invent. Math. 111 (1993), 349-373.

36. A. Rosenschon And V. SRinivas, The Griffiths group of the generic abelian 3-fold, in Proc. Int. Colloq. on Cycles, Motives and Shimura Varieties, Mumbai, TIFR Studies in Mathematics, Volume 21, pp. 449-467 (Narosa Publishing, 2010).

37. C. Schoen, Cyclic covers of $\mathbf{P}^{v}$ branched along $v+2$ hyperplanes and the generalized Hodge conjecture for certain abelian varieties, in Arithmetic of Complex Manifolds (Erlangen, 1988), Lecture Notes in Mathematics, Volume 1399, pp. 137-154 (Springer, Berlin, 1989).

38. C. Schoen, An integral analog of the Tate conjecture for one-dimensional cycles on varieties over finite fields, Math. Ann. 311 (1998), 493-500.

39. C. Schoen, The Chow group modulo $\ell$ for the triple product of a general elliptic curve, Asian J. Math. 4 (2000), 987-996.

40. C. Schoen, On certain exterior product maps of Chow groups, Math. Res. Lett. 7 (2000), $177-194$. 
41. C. Schoen, Complex varieties for which the Chow group $\bmod n$ is not finite, J. Algebraic Geom. 11 (2002), 41-100.

42. A. Suslin, Higher Chow groups and etale cohomology, in Cycles, Transfers, and Motivic Homology Theories, Annals of Mathematical Studies, Volume 143, pp. 239-254 (Princeton University Press, 2002).

43. C. Soulé And C. Voisin, Torsion cohomology classes and algebraic cycles on complex projective manifolds, Adv. Math. 198 (2005), 107-127.

44. A. Suslin And V. Voevodsky, Singular homology of abstract algebraic varieties, Invent. Math. 123 (1996), 61-94.

45. A. Suslin and V. Voevodsky, Bloch-Kato conjecture and motivic cohomology with finite coefficients, in The Arithmetic and Geometry of Algebraic Cycles (Banff, AB, 1998), NATO Science Series C: Mathematics, Physics and Science, Volume 548, pp. 117-189.

46. J. TATE, On the conjectures of Birch and Swinnerton-Dyer and a geometric analog, in Séminaire Bourbaki, 9, Exp. No. 306, pp. 415-440 (Soc. Math. France, 1965).

47. J. TATE, Relations between $K_{2}$ and Galois cohomology, Invent. Math. 36 (1976), 257-274.

48. J. TATE, Conjectures on algebraic cycle in $\ell$-adic cohomology, in Motives, Seattle, WA, 1991, Proceedings of Symposia in Pure Mathematics, Volume 55, pp. 71-83 (American Mathematical Society).

49. R. W. Thomason, A finiteness condition equivalent to the Tate conjecture over $\mathbf{F}_{q}$, in Algebraic K-Theory and Algebraic Number Theory, Honululu, HI, 1987, Contemporary Mathematics, Volume 83, pp. 385-392.

50. B. Totaro, Torsion algebraic cycles and complex cobordism, J. Amer. Math. Soc. 10 (1997), 467-493.

51. V. Voevodsky, Motivic cohomology with Z/2-coefficients, Publ. Math. Inst. Hautes Études Sci. 98 (2003), 59-104.

52. V. Voevodsky, On motivic cohomology with $\mathbf{Z} /$ l-coefficients, Ann. of Math. (2) $\mathbf{1 7 4}$ (2011), 401-438.

53. C. Voisin, On integral Hodge classes on uniruled and Calabi-Yau manifolds, in Moduli Spaces and Arithmetic Geometry, Advanced Studies in Pure Mathematics, Volume 45, pp. 43-73. (2006).

54. C. Voisin, Remarks on curve classes on rationally connected varieties, in A Celebration of Algebraic Geometry, Clay Math. Proc. 18, pp. 591-599 (American Mathematical Society, Providence, RI, 2013).

55. C. Voisin, Degree 4 unramified cohomology with finite coefficients and torsion codimension 3 cycles, in Geometry and Arithmetic, Schiermonnikoog, EMS Congress Report, pp. 347-368 (European Mathematical Society, 2010).

56. C. Weibel, The norm residue isomorphism theorem, J. Topol. 2 (2009), 346-372. 